\title{
OPEN The effects of anticholinergic medications on cognition in children: a systematic review and meta-analysis
}

\author{
Erica Ghezzi ${ }^{1,4}$, Michelle Chan ${ }^{1,4}$, Lisa M. Kalisch Ellett ${ }^{2}$, Tyler J. Ross ${ }^{1}$, Kathryn Richardson ${ }^{3}$, \\ Jun Ni Ho${ }^{2}$, Dayna Copley ${ }^{1}$, Claire Steele ${ }^{1}$ \& Hannah A. D. Keage ${ }^{1 \bowtie}$
}

Cognitive side effects of anticholinergic medications in older adults are well documented. Whether these poor cognitive outcomes are observed in children has not been systematically investigated. We aimed to conduct a systematic review and meta-analysis on the associations between anticholinergic medication use and cognitive performance in children. Systematic review was conducted using Medline, PsychInfo, and Embase, identifying studies testing cognitive performance relative to the presence versus absence of anticholinergic medication(s) in children. We assessed effects overall, as well as relative to drug class, potency (low and high), cognitive domain, and duration of administration. The systematic search identified 46 articles suitable for meta-analysis. For the most part, random effects meta-analyses did not identify statistically significant associations between anticholinergic exposure and cognitive performance in children; the one exception was a small effect of anticholinergic anti-depressants being associated with better cognitive function (Hedges' $g=0.24$, $95 \% \mathrm{Cl} 0.06-0.42, p=0.01$ ). Anticholinergic medications do not appear to be associated with poor cognitive outcomes in children, as they do in older adults. The discrepancy in findings with older adults may be due to shorter durations of exposure in children, differences in study design (predominantly experimental studies in children rather than predominantly epidemiological in older adults), biological ageing (e.g. blood brain barrier integrity), along with less residual confounding due to minimal polypharmacy and comorbidity in children.

Anticholinergic medications are commonly prescribed ${ }^{1-3}$ yet a growing body of evidence has demonstrated that their use is associated with a higher risk of incident cognitive impairment ${ }^{4-6}$. This literature has been reviewed multiple times in older adults, whereby anticholinergic medications have been consistently associated with cognitive decline and dementia ${ }^{7-9}$. There has been no systematic synthesis of the cognitive effects of anticholinergic medications in children.

There are few population-based studies that have assessed the extent to which children are exposed to anticholinergic medicines ${ }^{1}$. Most studies examining anticholinergic medicines in children have focussed on the use of medicine classes for specific indications, for example, asthma or overactive bladder, rather than providing population-based estimates for the use of anticholinergic medicines like the studies in older adults. Approximately $11 \%$ of Australian children have a current diagnosis of asthma ${ }^{10}$ and up to $20 \%$ of children experience bedwetting ${ }^{11}$ so there is potential for a high prevalence of use of anticholinergic medicines to treat these conditions in children. One population based study from Slovenia reported that $20 \%$ of children using prescription medicines were dispensed anticholinergic medicines, most commonly antihistamines ${ }^{1}$.

Anticholinergic medications refer to a broad class of medicines which block the neurotransmitter acetylcholine ${ }^{12}$. These medications are used in the treatment of many conditions such as depression, vertigo, asthma, cardiac arrhythmias and incontinence. High potency anticholinergic medications appear to most detrimentally affect cognition in older adults (as compared to low potency) ${ }^{13}$. Further, the class of anticholinergic medication differentially associates with cognitive decline in late-life, with anti-depressants (amitriptyline,

${ }^{1}$ Justice and Society, University of South Australia, GPO BOX 2741, Adelaide 5000, Australia. ${ }^{2}$ Clinical and Health Sciences, University of South Australia, Adelaide, Australia. ${ }^{3}$ Norwich Medical School, University of East Anglia, Norwich, UK. ${ }^{4}$ These authors contributed equally: Erica Ghezzi and Michelle Chan. ${ }^{\varpi}$ email: Hannah.Keage@ unisa.edu.au 
dosulepin, paroxetine), urologicals (oxybutynin, tolterodine), and antiparkinsonian drugs showing the strongest associations with incident dementia ${ }^{4}$. Neurobiologically, the cholinergic system primarily mediates attentional processes $^{14-17}$ and therefore could be expected to be primarily impaired by anticholinergic medications, although cognitive domain specific effects have not been investigated.

The current study aims to quantitively synthesise the literature on associations between anticholinergic medications and cognitive performance in children. Findings from this review will inform medical practitioners of any risks (or lack thereof) associated with anticholinergic use in children, and subsequently help to inform the safe prescribing of anticholinergics. It is critical to identify whether anticholinergics should be prescribed with restraint in children. We hypothesise that in children (1) exposure to anticholinergic medications will be significantly negatively associated with performance on cognitive tests, and that associations will be strongest for (2) antidepressant and urological drug classes (as compared to other drug classes), (3) high-potency anticholinergics (as compared to low-potency), (4) those exposed long-term (as opposed to short-term) and (5), within the cognitive domain of attention.

\section{Methods}

Search strategy. This study adhered to the Preferred Reporting Items for Systematic Reviews and MetaAnalyses (PRISMA) guidelines (see Supplementary Table 1 for PRISMA Checklist) ${ }^{18,19}$. A systematic literature search was conducted in December 2019 using the electronic databases Medline, PsychInfo, and Embase. The search strategy used a combination of keywords for anticholinergic medications (see Supplementary Material), cognition terms (cognit ${ }^{\star}$ OR neuropsych ${ }^{\star}$ OR learn ${ }^{\star}$ OR memory OR "executive function" OR "executive functions") and demographic terms (children OR childhood OR youth ${ }^{\star}$ OR teen ${ }^{\star}$ ). No published review protocol exists for the current study.

Anticholinergic medications were defined as medicines with clinically significant anticholinergic properties as listed in a systematic review by Duran et al. ${ }^{12}$. Medications assessed by the Duran systematic review to be of either high or low anticholinergic potency, but not ambiguous potency, were included. Studies were screened and assessed for eligibility by two independent reviewers, first by title and abstract, then by full text, according to inclusion and exclusion criteria described below (MC, TJR, DC, CS and JNH). Any conflicts were resolved through consensus.

Inclusion and exclusion criteria. Studies of either within- or between-groups design were included if they reported at least one cognitive outcome for both children exposed and unexposed to anticholinergic medications; reported data for a sample of children ( $<18$ years old); were published in English; and were published in peer-reviewed journal articles. Studies from all publication years were accepted. "On" medication participants included children exposed to at least one anticholinergic medication. "Off" medication participants included matched controls unexposed to any other medication, participants treated with placebo, participants undergoing withdrawal from the medication, or the baseline measurements of the exposed group. To be eligible for inclusion, studies needed to report cognitive outcomes based on objective cognitive measures; subjective behavioural reports were not included (e.g. self, parent or teacher reports of cognitive functioning). Studies were excluded if the control group did not share the same disorder or symptom (i.e. healthy control group) of the experimental group. Studies which only compared the effects of anticholinergic medication versus non-anticholinergic medication, rather than anticholinergic medication versus no medication, were excluded. Studies were also excluded if they involved non-human (animal) participants; if they assessed in-utero anticholinergic exposure; or if they were a case report, case series, thesis or conference abstract.

Data extraction. Data were extracted from eligible studies independently by one reviewer (EG, MC, TJR) and then checked by a second reviewer, with any discrepancies resolved through discussion or checked again (by a third reviewer). Extracted data include country of publication, study design, sample size (and number of male/ female participants), age, diagnoses of sample, name of medication, duration of administration, and cognitive domains assessed. The extracted medication name was then classified by potency and drug class by an academic pharmacist (LE). Data required for meta-analysis were also extracted. This included any data for which an effect size (standardised mean difference) could be calculated for differences between on and off medication groups (e.g., means and standard deviations, Cohen's d and confidence intervals (CIs), sample size and correlation statistic, means and correlation statistic, or means and p-value).

Quality assessment. A quality assessment tool was developed for this study, adapted from a critical appraisal tool for randomised controlled trials from the Joanna Briggs Institute ${ }^{20}$, see Supplementary MaterialQuality Assessment Tool. The Joanna Briggs Institute is a highly regarded organization with recommended ${ }^{21}$ and well-used critical appraisal checklists ${ }^{22-24}$. The quality assessment tool comprised an eight-point checklist. All studies were screened using this tool by two independent reviewers (MC and TJR) and any conflicts in scoring were resolved through discussion.

Statistical approach. Some included studies reported data for both within- and between-groups designs. For example, they may include two groups: one that experiences a period of on and off medication, and one non-medicated control group. In these cases, the between-group design (i.e. medication versus control) was preferentially selected in order to minimise the effect of cognitive development (over time). Where one study reported both within- and between-group comparisons for two distinct participant samples (i.e. one group both on and off medication, along with a second group on medication and a third no-medication control group) both within- and between-groups data were extracted. In cases where one study reported both (within and between) 
comparisons over multiple time-points, within-groups data were extracted for any time-points where betweengroups data were unavailable.

All outcome measures were standardised using Hedges' $g$ for difference between on- and off-medication groups. A positive Hedges' $g$ represents a better cognitive score for the on-medication group compared to the off-medication group, regardless of the direction of the original cognitive test. Small, medium, and large effect sizes were classified using the Hedges and Olkin ${ }^{25}$ method, as $0.20,0.50$, and 0.80 respectively. Comprehensive Meta-Analysis software (version 3) was used to calculate effect sizes, where calculations of Hedge's $g$ are dependent on study design (within- or between-groups). Statistical analyses were conducted using the meta package ${ }^{26}$ for R (Version 4.0.2). Dependency was present in analyses due to included studies reporting multiple cognitive outcomes or time-points for follow-up based on the same, or largely overlapping, participant samples. This was accounted for by averaging across effect sizes within studies, so one effect size was used per study within each analysis. The data and script associated with this analysis are publicly available (https://github.com/ericaghezz i/anticholinergic_med_metaanalysis).

Outcomes across studies were pooled using a random-effects model. The commonly used DerSimonian and Laird ${ }^{27}$ estimator of between-study variance has been criticised due to its propensity to underestimate true between-study variance, leading to narrow CIs and potential false-positive estimations ${ }^{28,29}$. Hence, we followed the recommendation of Veroniki et al. ${ }^{30}$ and employed the Paule and Mandel ${ }^{31}$ method, which has been shown to be less biased ${ }^{29,32}$ when estimating between-study variance. Sensitivity analyses revealed no substantial differences in outcomes when analyses were run using common between-groups estimators. The Hartung-Knapp method for random effects meta-analysis ${ }^{33,34}$ was also applied to all analyses. A result was considered statistically significant when $p<0.05$. We considered this an exploratory study and did not correct for multiple comparisons. Between-study variance was quantified using $\tau^{2}$. The proportion of between-study heterogeneity out of total variance was assessed using the $\mathrm{I}^{2}$ statistic. Values of $\mathrm{I}^{2}$ were classified as low $(25 \%)$, moderate $(50 \%)$, or high $(75 \%)^{35}$.

Subgroup analysis. Subgroup analyses were stratified by anticholinergic potency, cognitive domain, drug class, and duration of medication administration. Anticholinergic potency was classified as low or high according to Durán et al. ${ }^{12}$. Cognitive domain was based on Lezak et al. ${ }^{36}$ : attention, psychomotor functioning, concept formation and reasoning, perception, memory, executive function, language, and intelligence. The anticholinergic drugs administered were categorised by class as antiepileptics (WHO Anatomical Therapeutic Chemical code N03), antiparkinsonian medicines (N04B), antipsychotics (N05A), antidepressants (N06A), respiratory medicines (R), opioid analgesics (N02A), or urological medicines (G04B). Only one study ${ }^{37}$ reported results based on an antiparkinsonian anticholinergic, so subgroup meta-analysis of this medication class was not conducted (note: the study was included in the overall meta-analysis). Total volume of exposure or dose has been shown to be important in assessing risk of cognitive impairment associated with use of anticholinergic medicines in adults; however, dose was inconsistently reported, or not reported at all, in many of the studies included in the meta-analysis. Duration of exposure, which was consistently reported in the studies, was therefore analysed. Duration of medication administration was categorised as either (1) current and long-term (>1-month), (2) current and acute ( $\leq 1$-month) and (3) historical administration. Each subgroup analysis was based on a random-effects model, where calculations of within-subgroup variance and comparisons between subgroups were both made using a random-effects model. Fixed effects comparisons of differences between subgroups were not made due to the risk of false positives ${ }^{38}$. The $\mathrm{Q}$ statistic was calculated as a test of between subgroups differences.

Publication bias. Funnel plots of effect size versus standard error for the primary outcome were visually examined for symmetry to assess for bias across studies due to the small-study effect ${ }^{39}$. As the whole metaanalysis contained at least 10 studies, small-study effect was formally tested using Egger's test of the intercept ${ }^{40}$. If evidence of asymmetry was found (one-tailed $p<0.1$ on the Egger's test), Duval and Tweedie's ${ }^{41}$ trim and fill method would have been used to quantify the magnitude of potential bias.

\section{Results}

Summary of studies. A total of 7,645 articles were identified, of which 6,283 were screened by title and abstract following duplicate removal. Full-text review was conducted on 323 articles, and 46 of these were included for final review and meta-analysis (Fig. 1). The 46 included studies were published across 6 decades, with $1,2,7,10,13$, and 13 studies published in ascending decades from the 1960s. Of the included studies, 37 were conducted in developed countries, 7 in developing countries, and 2 included children from both developing and developed countries (classified according to the $\mathrm{UN}^{42}$ ). For a complete overview of the characteristics of included studies, see Table 1 .

Overall cognition. Overall, the 46 studies included reported a total of 536 effect sizes. The pooled effect size of the difference between cognition on and off medication across the 46 studies was negligible and non-significant $(g=0.05,95 \% \mathrm{CI}-0.02$ to $0.11, p=0.16$; see Fig. 2$)$, with no heterogeneity between studies $\left(\tau^{2}=0, I^{2}=0 \%\right.$, $\mathrm{Q}=42.36$ ). The funnel plot did not reveal significant asymmetry (Egger's intercept $=-0.5, p=0.14$; see Fig. 3 ).

Subgroup analyses. Pooled estimates for subgroup analyses by anticholinergic drug class, potency, length of administration and cognitive domain are presented in Table 2. The number of studies within individual subanalyses ranged from 2 to 37 . Varying levels of heterogeneity were present across analyses, ranging from null to high ( $\tau^{2}$ range: $0-0.13, \mathrm{I}^{2}$ range: $\left.0-76.2, \mathrm{Q}=0.18-54.70\right)$.

No significant differences between subgroups were revealed through a test of between-subgroup differences using the random-effects model (see Table 2). The pooled effect size for cognitive outcomes on antidepressant medications was small and statistically significant (see Table 2 and Fig. 4), with negligible heterogeneity between 


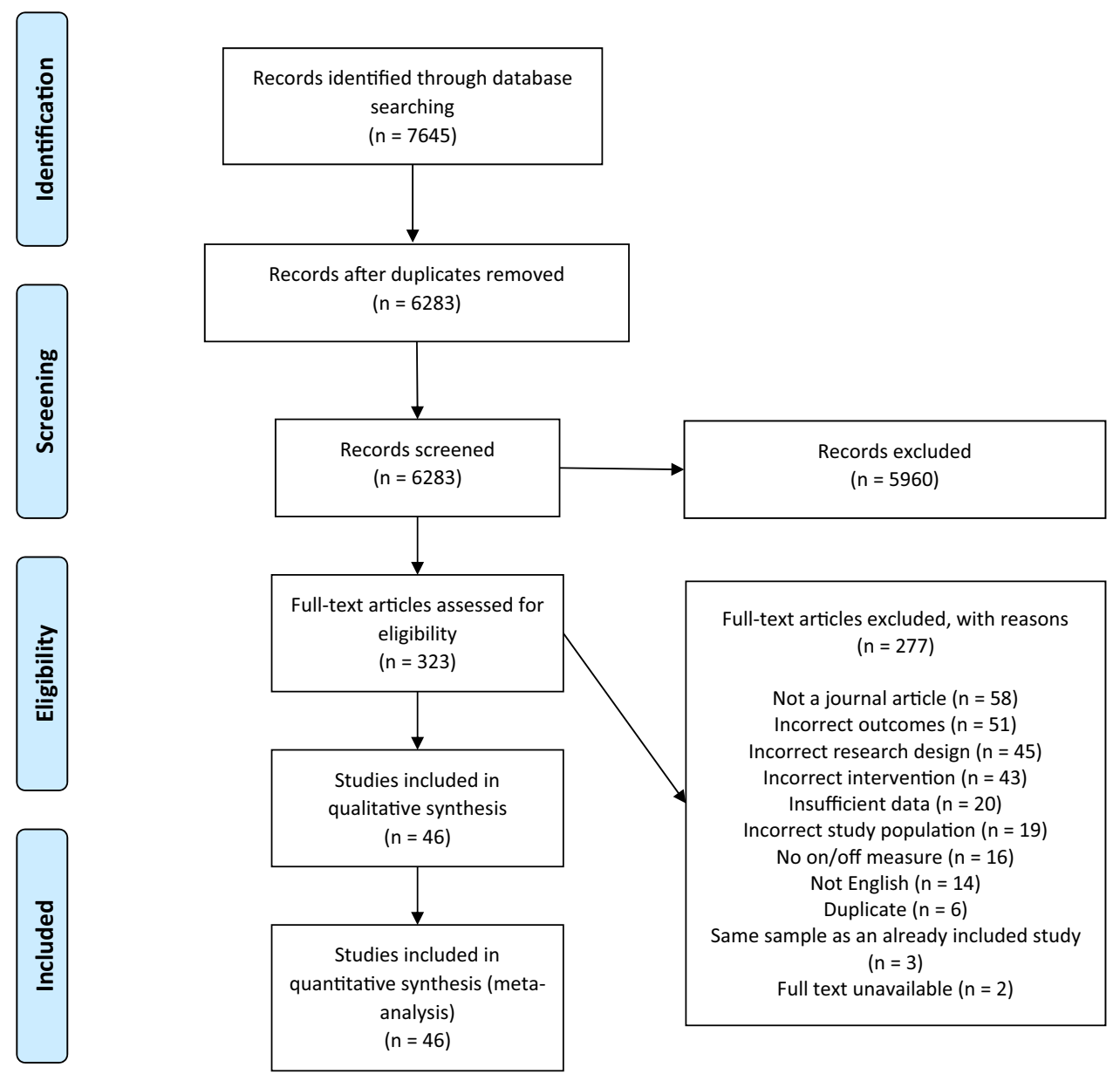

Figure 1. PRISMA flow diagram of the article selection and screening process. The databases searched were Medline, PsychInfo, and Embase.

studies $\left(\tau^{2}=0, I^{2}=13.2 \%, \mathrm{Q}=6.91\right)$. Notably, this effect was not significant in a sensitivity analysis (Supplementary Table 3 ) which included only studies of high quality. Pooled estimates were non-significant across the remaining anticholinergic drug class (see Fig. 4), potency (see Fig. 5), length of administration (see Fig. 6), and cognitive domain (see Fig. 7) subgroup analyses. All null results were replicated within the sensitivity analysis of high-quality studies, except the memory cognitive domain analysis, which had a small positive significant effect $(g=0.09,95 \%$ CI $0.01-0.17, p=0.02)$.

\section{Discussion}

We quantified the effects of anticholinergic medications on cognition in children systematically across the literature. We report that, unlike older adult samples ${ }^{7-9}$, anticholinergic medications are not associated with cognitive impairments in children. This finding was regardless of the classification approach used: drug class, potency, duration of use, and cognitive domain. The discrepancy between child and older adult samples may be due to shorter lengths of exposure in children, higher rates of polypharmacy in older adults ${ }^{88}$, residual confounding, study design, or biological ageing processes.

Older adults have the opportunity for years or decades of anticholinergic exposure ${ }^{88}$, with polypharmacy common, whereas studies included here from child samples typically had short exposure durations ( 6 months or less in most studies) and little polypharmacy. It may be that the detrimental effect of anticholinergic medications on cognition in late-adulthood is driven by long exposure and polypharmacy ${ }^{89,90}$, factors not observed in children. Further, in late-life, the class of antidepressant appears to differentially affect cognition, with anti-depressants, urologicals, and antiparkinsonian drugs showing the strongest associations with incident dementia risk ${ }^{4}$. We did not see this pattern of effects in children. It may be that duration of exposure and polypharmacy again drives this difference, however residual confounding in late-life samples cannot be ruled out. It may be that incontinence and mood symptoms, for which anticholinergic medications are prescribed, are early clinical indicators of dementiarelated neuropathologies ${ }^{4}$ (which accrue decades prior to a dementia diagnosis ${ }^{91}$ ) and that early, undiagnosed dementia is driving the associations between use of anticholinergic medicines and poor cognition in adults.

Interestingly, all study designs included in this review were experimental, whereas those included in reviews of older adults are typically longitudinal epidemiological cohort studies ${ }^{7-9}$. Standards of reporting cognitive 


\begin{tabular}{|c|c|c|c|c|c|c|c|c|c|c|c|c|}
\hline \multirow[b]{2}{*}{ Author } & \multirow[b]{2}{*}{ Year } & \multirow[b]{2}{*}{ Country } & \multirow[b]{2}{*}{ Design } & \multicolumn{3}{|l|}{ Sample } & \multicolumn{5}{|c|}{ Anticholinergic medication } & \multirow[b]{2}{*}{$\begin{array}{l}\text { Cognitive } \\
\text { domain }(s)\end{array}$} \\
\hline & & & & $\mathbf{N}(\mathbf{M} / \mathbf{F})$ & $\begin{array}{l}\text { Age in } \\
\text { years }^{*}\end{array}$ & Diagnoses & Name & Potency & $\begin{array}{l}\text { Class/ } \\
\text { function }\end{array}$ & \begin{tabular}{|l|}
$\begin{array}{l}\text { Length of } \\
\text { administration }\end{array}$ \\
\end{tabular} & $\begin{array}{l}\text { Medication } \\
\text { duration }\end{array}$ & \\
\hline $\begin{array}{l}\text { Aldenkamp } \\
\text { et al. }{ }^{43}\end{array}$ & 1993 & Sweden & $\begin{array}{l}\text { NRCT } \\
\text { (Within) }\end{array}$ & $83(47 / 36)$ & $12.8(2.4)$ & Epilepsy & $\begin{array}{l}\text { Carbamaz- } \\
\text { epine }\end{array}$ & Low & Antiepileptic & Long & $>1$ year & Att., PM \\
\hline Aman et al. ${ }^{44}$ & 2008 & USA & $\begin{array}{l}\text { RCT } \\
\text { (Between**) }\end{array}$ & $38(29 / 9)$ & $9.4(3.0)$ & $\begin{array}{l}\text { Autism + Severe } \\
\text { behavioural distur- } \\
\text { bance }\end{array}$ & Risperidone & Low & $\begin{array}{l}\text { Antipsy- } \\
\text { chotic }\end{array}$ & Acute, Long & $\begin{array}{l}4 \text { weeks, } 8 \\
\text { weeks }\end{array}$ & $\begin{array}{l}\text { Att., CF +R, } \\
\text { Mem., Perc., } \\
\text { PM, }\end{array}$ \\
\hline Aman et al. ${ }^{45}$ & 2009 & USA & $\begin{array}{l}\text { RCT (Crosso- } \\
\text { ver) }\end{array}$ & $16(14 / 2)$ & $8.6(2.6)$ & $\begin{array}{l}\text { DBD/ADHD/High- } \\
\text { functioning autism }\end{array}$ & Risperidone & Low & $\begin{array}{l}\text { Antipsy- } \\
\text { chotic }\end{array}$ & Acute & 2 weeks & Att., PM \\
\hline $\begin{array}{l}\text { Barrickman } \\
\text { et al. }{ }^{46}\end{array}$ & 1991 & USA & $\begin{array}{l}\text { NRCT } \\
\text { (Within) }\end{array}$ & $19(16 / 3)$ & $11.0(2.3)$ & ADHD & Fluoxetine & Low & \begin{tabular}{|l|}
$\begin{array}{l}\text { Antidepres- } \\
\text { sant }\end{array}$ \\
\end{tabular} & Long & 6 weeks & Att., EF, Int \\
\hline Beers et al. ${ }^{37}$ & 2005 & USA & $\begin{array}{l}\text { RCT } \\
\left(\text { Between }{ }^{* *}\right)\end{array}$ & 13 & $11.9(3.0)$ & TBI & Amantadine & Low & \begin{tabular}{|l|}
$\begin{array}{l}\text { Antiparkin- } \\
\text { sonian }\end{array}$ \\
\end{tabular} & Long & 12 weeks & $\begin{array}{l}\text { Att., } C F+R \text {, } \\
\text { EF }\end{array}$ \\
\hline $\begin{array}{l}\text { Bender and } \\
\text { Milgrom }^{47}\end{array}$ & 2004 & USA & RCT (Between) & 60 & {$[8-17]$} & SAR & Loratadine & Low & Respiratory & Acute & 2 weeks & Att., Mem \\
\hline Bender et al. ${ }^{48}$ & 1991 & USA & $\begin{array}{l}\text { NRCT } \\
\text { (Between) }\end{array}$ & 63 & $11.7(2.1)$ & Asthma & Theophylline & Low & Respiratory & Acute & $\begin{array}{l}1 \text { week, } 1 \\
\text { month, } 3 \\
\text { months, } 6 \\
\text { months }\end{array}$ & Att \\
\hline Carlson et al. ${ }^{49}$ & 1992 & USA & $\begin{array}{l}\text { NRCT } \\
\text { (Crossover) }\end{array}$ & $11(8 / 3)$ & $8.7(2.4)$ & $\begin{array}{l}\mathrm{CD} \text { with manic } \\
\text { symptoms/CD } \\
\text { with family BPD } \\
\text { history/Aggressive } \\
\text { behaviour }\end{array}$ & Lithium & Low & $\begin{array}{l}\text { Antipsy- } \\
\text { chotic }\end{array}$ & Acute, Long & $\begin{array}{l}4 \text { weeks, } 8 \\
\text { weeks }\end{array}$ & $\begin{array}{l}\text { Att., } \mathrm{EF}+\mathrm{R} \text {, } \\
\text { Mem }\end{array}$ \\
\hline Chen et al. ${ }^{50}$ & 2001 & Taiwan & $\begin{array}{l}\text { NRCT } \\
\text { (Within) }\end{array}$ & $25(13 / 12)$ & $11.2(2.0)$ & Epilepsy & $\begin{array}{l}\text { Carbamaz- } \\
\text { epine }\end{array}$ & Low & Antiepileptic & Long & $>1$ year & Int \\
\hline de Graaf et al. ${ }^{51}$ & 2011 & Netherlands & RCT (Between) & $90(51 / 39)$ & $\begin{array}{l}<3 \mathrm{~d} \text { at } \\
\text { exposure; } 5 \\
\text { at follow up }\end{array}$ & Pain & Morphine & Low & $\begin{array}{l}\text { Opioid } \\
\text { analgesic }\end{array}$ & History & NR & Int., PM \\
\hline de Graaf et al. ${ }^{52}$ & 2013 & Netherlands & RCT (Between) & $89(56 / 33)$ & $\begin{array}{l}<3 d \text { at } \\
\text { exposure; } \\
8-9 \text { at } \\
\text { follow-up }\end{array}$ & Pain & Morphine & Low & $\begin{array}{l}\text { Opioid } \\
\text { analgesic }\end{array}$ & History & NR & $\begin{array}{l}\text { Att., CF + R, } \\
\text { EF, Int., PM }\end{array}$ \\
\hline Donati et al. ${ }^{53}$ & 2007 & $\begin{array}{l}\text { Europe (7 } \\
\text { countries) }\end{array}$ & RCT (Within) & $83(37 / 46)$ & $10[6-16]$ & Partial seizures & $\begin{array}{l}\text { Oxcarbaz- } \\
\text { epine, } \\
\text { Carbamaz- } \\
\text { epine }\end{array}$ & Low & Antiepileptic & Long & 6 months & $\begin{array}{l}\text { Att., Mem, } \\
\text { Perc., PM }\end{array}$ \\
\hline Erickson et al. ${ }^{54}$ & 1984 & USA & RCT (Within) & 11 & $\begin{array}{l}14.2 \\
(12.9-18.6)\end{array}$ & $\begin{array}{l}\text { Schizophrenia/ } \\
\text { Schizophreniform } \\
\text { disorder }\end{array}$ & \begin{tabular}{|l|} 
Thiori- \\
dazine, \\
Thiothixene
\end{tabular} & High & $\begin{array}{l}\text { Antipsy- } \\
\text { chotic }\end{array}$ & Long & 35 days & Att \\
\hline Eun et al..$^{55}$ & $2012 \mathrm{a}$ & South Korea & RCT (Within) & $41(24 / 17)$ & $8.3(2.1)$ & Epilepsy & $\begin{array}{l}\text { Carbamaz- } \\
\text { epine }\end{array}$ & Low & Antiepileptic & Long & 32 weeks & Int \\
\hline Eun et al. ${ }^{56}$ & $2012 b$ & South Korea & $\begin{array}{l}\text { NRCT } \\
\text { (Within) }\end{array}$ & $168(98 / 70)$ & $8.4(2.7)$ & Epilepsy & $\begin{array}{l}\text { Oxcarbaz- } \\
\text { epine }\end{array}$ & Low & Antiepileptic & Long & 26-32 weeks & $\begin{array}{l}\text { Att., Int., } \\
\text { CF + R, Lan., } \\
\text { PM }\end{array}$ \\
\hline Farmer et al. ${ }^{57}$ & 2017 & USA & RCT (Between) & $165(128 / 3)$ & $8.9(2.0)$ & $\begin{array}{l}\text { ADHD+ Severe } \\
\text { physical aggression }\end{array}$ & Risperidone & Low & $\begin{array}{l}\text { Antipsy- } \\
\text { chotic }\end{array}$ & Acute & 3 weeks & Att \\
\hline Ferguson et al. ${ }^{58}$ & 2012 & USA & RCT (Between) & $19(12 / 7)$ & $\begin{array}{l}\text { Neonate } \\
\text { exposure; } \\
6.2(0.3) \text { at } \\
\text { follow-up }\end{array}$ & Pain & Morphine & Low & $\begin{array}{l}\text { Opioid } \\
\text { analgesic }\end{array}$ & History & $\leq 14$ days & $\begin{array}{l}\text { Att., } \mathrm{CF}+\mathrm{R} \text {, } \\
\text { Int., Lan }\end{array}$ \\
\hline Forsythe et al. ${ }^{59}$ & 1991 & UK & RCT (Within) & $14(7 / 7)$ & 10 & Epilepsy & $\begin{array}{l}\text { Carbamaz- } \\
\text { epine }\end{array}$ & Low & Antiepileptic & Acute, Long & $\begin{array}{l}1 \text { month, } 6 \\
\text { months, } 12 \\
\text { months }\end{array}$ & Att., Mem \\
\hline Freibergs et al. ${ }^{60}$ & 1968 & Canada & $\begin{array}{l}\text { RCT } \\
\left(\text { Between }^{* *}\right)\end{array}$ & $36(36 / 0)$ & $8.7(6-12)$ & Hyperactivity & $\begin{array}{l}\text { Chlorprom- } \\
\text { azine }\end{array}$ & High & $\begin{array}{l}\text { Antipsy- } \\
\text { chotic }\end{array}$ & Long & 74.8 days & $\mathrm{CF}+\mathrm{R}$ \\
\hline $\begin{array}{l}\text { Giramonti } \\
\text { et al. }{ }^{61}\end{array}$ & 2008 & USA & $\begin{array}{l}\text { RCT (Crosso- } \\
\text { ver) }\end{array}$ & $14(9 / 5)$ & $7.7(2.0)$ & Incontinence & $\begin{array}{l}\text { Oxybutynin, } \\
\text { Tolterodine }\end{array}$ & High & Urological & Acute & 2 weeks & Att., Mem \\
\hline $\begin{array}{l}\text { Gualtieri and } \\
\text { Evans }{ }^{62}\end{array}$ & 1988 & USA & $\begin{array}{l}\text { RCT (Crosso- } \\
\text { ver) }\end{array}$ & $9(6 / 3)$ & $9.5(1.3)$ & ADHD & Imipramine & High & $\begin{array}{l}\text { Antidepres- } \\
\text { sant }\end{array}$ & Acute & 2-3 days & Att., PM \\
\hline Gualtieri et al. ${ }^{63}$ & 1991 & USA & $\begin{array}{l}\text { RCT (Crosso- } \\
\text { ver) }\end{array}$ & $12(11 / 1)$ & {$[6-12]$} & ADHD & Desipramine & High & $\begin{array}{l}\text { Antidepres- } \\
\text { sant }\end{array}$ & Acute & 2-3 days & $\begin{array}{l}\text { Att., Mem, } \\
\text { PM }\end{array}$ \\
\hline Gunther et al. ${ }^{64}$ & 2006 & Germany & $\begin{array}{l}\text { NRCT } \\
\text { (Within) }\end{array}$ & $23(21 / 2)$ & $11.9(2.1)$ & $\mathrm{ADHD}+\mathrm{DBD}$ & Risperidone & Low & $\begin{array}{l}\text { Antipsy- } \\
\text { chotic }\end{array}$ & Acute & 4 weeks & Att., EF \\
\hline Jung et al. ${ }^{65}$ & 2015 & South Korea & RCT (Within) & 40 & {$[4-16]$} & Epilepsy & $\begin{array}{l}\text { Carbamaz- } \\
\text { epine }\end{array}$ & Low & Antiepileptic & Long & 52 weeks & Int \\
\hline Klein $^{66}$ & 1990 & USA & $\begin{array}{l}\text { RCT (Within } \\
\text { \& Between) }\end{array}$ & $36(33 / 3)$ & $8.5(1.6)$ & $\begin{array}{l}\text { ADHD + Hyper- } \\
\text { activity }\end{array}$ & Thioridazine & High & $\begin{array}{l}\text { Antipsy- } \\
\text { chotic }\end{array}$ & Acute, Long & $\begin{array}{l}4 \text { weeks, } 12 \\
\text { weeks }\end{array}$ & $\begin{array}{l}\text { Att., CF + R, } \\
\text { EF, Int., Lan., } \\
\text { Mem., PM }\end{array}$ \\
\hline Kwon et al. ${ }^{67}$ & 2013 & South Korea & $\begin{array}{l}\text { NRCT } \\
\left(\text { Between }^{* *}\right)\end{array}$ & $29(17 / 15)$ & $8.4(2.3)$ & Epilepsy & $\begin{array}{l}\text { Oxcarbaz- } \\
\text { epine }\end{array}$ & Low & Antiepileptic & Long & 6 months & $\begin{array}{l}\text { Att., } \mathrm{CF}+\mathrm{R} \text {, } \\
\mathrm{EF} \text {, Int }\end{array}$ \\
\hline $\begin{array}{l}\text { O'Dougherty } \\
\text { et al. }{ }^{68}\end{array}$ & 1987 & USA & $\begin{array}{l}\text { NRCT } \\
\text { (Within) }\end{array}$ & $11(4 / 7)$ & $9.8(3.1)$ & Epilepsy & $\begin{array}{l}\text { Carbamaz- } \\
\text { epine }\end{array}$ & Low & Antiepileptic & Long & $\begin{array}{l}3 \text { weeks- } \\
10 \text { months }\end{array}$ & $\begin{array}{l}\text { Att., Mem, } \\
\text { PM }\end{array}$ \\
\hline Operto et al. ${ }^{69}$ & 2020 & Italy & $\begin{array}{l}\text { NRCT } \\
\text { (Within) }\end{array}$ & $46(16 / 20)$ & $9.8(2.3)$ & Epilepsy & $\begin{array}{l}\text { Oxcar- } \\
\text { bazepine, } \\
\text { Carbamaz- } \\
\text { epine }\end{array}$ & Low & Antiepileptic & Long & 9 months & Comp \\
\hline
\end{tabular}




\begin{tabular}{|c|c|c|c|c|c|c|c|c|c|c|c|c|}
\hline \multirow[b]{2}{*}{ Author } & \multirow[b]{2}{*}{ Year } & \multirow[b]{2}{*}{ Country } & \multirow[b]{2}{*}{ Design } & \multicolumn{3}{|l|}{ Sample } & \multicolumn{5}{|c|}{ Anticholinergic medication } & \multirow[b]{2}{*}{\begin{tabular}{|l|} 
Cognitive \\
domain(s)
\end{tabular}} \\
\hline & & & & $\mathbf{N}(\mathbf{M} / \mathbf{F})$ & \begin{tabular}{|l|} 
Age in \\
years*
\end{tabular} & Diagnoses & Name & Potency & $\begin{array}{l}\text { Class/ } \\
\text { function }\end{array}$ & $\begin{array}{l}\text { Length of } \\
\text { administration }\end{array}$ & $\begin{array}{l}\text { Medication } \\
\text { duration }\end{array}$ & \\
\hline Pandina et al. ${ }^{70}$ & 2009 & \begin{tabular}{|l|} 
Europe (6 \\
countries), \\
Israel, South \\
Africa \\
\end{tabular} & $\begin{array}{l}\text { RCT (Within } \\
\text { \& Between) }\end{array}$ & $284(248 / 36)$ & $10.8(2.9)$ & DBD & Risperidone & Low & $\begin{array}{l}\text { Antipsy- } \\
\text { chotic }\end{array}$ & Long & $\begin{array}{l}6 \text { weeks, } 6 \\
\text { months }\end{array}$ & Att., Mem \\
\hline Piccinelli et al. ${ }^{71}$ & 2010 & Italy & $\begin{array}{l}\text { NRCT } \\
\text { (Within) }\end{array}$ & $43(21 / 22)$ & $10.4(3.1)$ & Epilepsy & \begin{tabular}{|l}
$\begin{array}{l}\text { Carbamaz- } \\
\text { epine }\end{array}$ \\
\end{tabular} & Low & Antiepileptic & Long & 12 months & $\mathrm{CF}+\mathrm{R}, \mathrm{Int}$ \\
\hline Platt et al..$^{72}$ & 1981 & USA & $\begin{array}{l}\text { RCT } \\
\left(\text { Between**) }^{*}\right.\end{array}$ & $30(28 / 2)$ & \begin{tabular}{|l|}
9.0 \\
$(5.8-12.9)$ \\
\end{tabular} & $\mathrm{CD}$ & \begin{tabular}{|l|}
$\begin{array}{l}\text { Haloperidol, } \\
\text { Lithium }\end{array}$ \\
\end{tabular} & Low & $\begin{array}{l}\text { Antipsy- } \\
\text { chotic }\end{array}$ & Acute & 4 weeks & Att., EF \\
\hline Platt et al. $^{73}$ & 1984 & USA & $\begin{array}{l}\text { RCT } \\
\left(\text { Between }^{* *}\right)\end{array}$ & $61(57 / 4)$ & \begin{tabular}{|l|}
9.0 \\
$(5.2-12.9)$
\end{tabular} & $\mathrm{CD}$ & \begin{tabular}{|l}
$\begin{array}{l}\text { Haloperidol, } \\
\text { Lithium }\end{array}$ \\
\end{tabular} & Low & $\begin{array}{l}\begin{array}{l}\text { Antipsy- } \\
\text { chotic }\end{array} \\
\end{array}$ & Acute & 4 weeks & Att., EF \\
\hline $\begin{array}{l}\text { Rappaport } \\
\text { et al. }^{74}\end{array}$ & 1989 & USA & $\begin{array}{l}\text { RCT (Crosso- } \\
\text { ver) }\end{array}$ & $17(11 / 6)$ & {$[6-12]$} & Asthma & Theophylline & Low & Respiratory & Acute & 3.5 days & \begin{tabular}{|l} 
Att., EF, \\
Mem., PM
\end{tabular} \\
\hline Robles et al. ${ }^{75}$ & 2011 & Spain & RCT (Within) & $49(38 / 11)$ & $15.9(1.4)$ & Psychosis & $\begin{array}{l}\text { Quetiapine, } \\
\text { Olanzapine }\end{array}$ & Low & $\begin{array}{l}\text { Antipsy- } \\
\text { chotic }\end{array}$ & Long & 6 months & $\begin{array}{l}\text { Att., CF+R, } \\
\text { Comp., EF, } \\
\text { Mem., Perc., } \\
\text { PM }\end{array}$ \\
\hline Schlieper et al. ${ }^{76}$ & 1991 & Canada & $\begin{array}{l}\text { RCT (Crosso- } \\
\text { ver) }\end{array}$ & $31(21 / 10)$ & $9.8(1.6)$ & Asthma & Theophylline & Low & Respiratory & Acute & 10 days & Att., EF Mem \\
\hline $\begin{array}{l}\text { Seidel and } \\
\text { Mitchell177}\end{array}$ & 1999 & USA & $\begin{array}{l}\text { NRCT } \\
\text { (Crossover) }\end{array}$ & $10(6 / 4)$ & $9.7(2.0)$ & Epilepsy & $\begin{array}{l}\text { Carbamaz- } \\
\text { epine }\end{array}$ & Low & Antiepileptic & Long & \begin{tabular}{|l|}
2.2 \\
months-2.1 \\
years
\end{tabular} & $\begin{array}{l}\text { Att., CF + R, } \\
\text { Int., Lan., } \\
\text { Mem., PM }\end{array}$ \\
\hline Shehab et al. ${ }^{78}$ & 2016 & Lebanon & $\begin{array}{l}\text { NRCT } \\
\text { (Within) }\end{array}$ & $24(8 / 16)$ & $14.8(1.6)$ & MDD & Fluoxetine & Low & $\begin{array}{l}\text { Antidepres- } \\
\text { sant }\end{array}$ & Long & $\begin{array}{l}\begin{array}{l}6 \text { weeks, } 12 \\
\text { weeks }\end{array} \\
\end{array}$ & Att., EF \\
\hline Sommer et al. ${ }^{79}$ & 2005 & USA & $\begin{array}{l}\text { NRCT } \\
\left.\text { (Between }{ }^{* *}\right)\end{array}$ & $25(11 / 14)$ & $7.2(1.8)$ & Incontinence & Oxybutynin & High & Urological & Acute & 4 weeks & Att., Mem \\
\hline $\begin{array}{l}\text { Stevenson } \\
\text { et al. }{ }^{80}\end{array}$ & 2002 & \begin{tabular}{|l|} 
Europe (12 \\
countries), \\
Brazil, \\
Canada
\end{tabular} & RCT (Between) & 165 & 2.92 & Dermatitis & Cetrizine & Low & Respiratory & Long & 8 weeks & Comp \\
\hline Tonnby et al. ${ }^{81}$ & 1994 & Sweden & $\begin{array}{l}\text { NRCT } \\
\text { (Within) }\end{array}$ & $100(56 / 44)$ & $12.5(2.1)$ & Epilepsy & \begin{tabular}{|l|}
$\begin{array}{l}\text { Carbamaz- } \\
\text { epine }\end{array}$
\end{tabular} & Low & Antiepileptic & Long & \begin{tabular}{|l|}
$\begin{array}{l}\text { Approx. } 3.7 \\
\text { years }\end{array}$ \\
\end{tabular} & $\begin{array}{l}\text { Att., Mem., } \\
\text { PM }\end{array}$ \\
\hline Troost et al. ${ }^{82}$ & 2006 & Netherlands & RCT (Within) & $24(22 / 2)$ & $9.3(2.6)$ & PDD & Risperidone & Low & $\begin{array}{l}\text { Antipsy- } \\
\text { chotic }\end{array}$ & Acute, Long & $\begin{array}{l}4 \text { weeks, } 8 \\
\text { weeks, } 24 \\
\text { weeks }\end{array}$ & Att \\
\hline $\begin{array}{l}\text { Tzitiridou } \\
\text { et al. }{ }^{83}\end{array}$ & 2005 & Greece & $\begin{array}{l}\text { NRCT } \\
\text { (Within) }\end{array}$ & $70(45 / 25)$ & $8.4(1.2)$ & Epilepsy & \begin{tabular}{|l|}
$\begin{array}{l}\text { Oxcarbaz- } \\
\text { epine }\end{array}$ \\
\end{tabular} & Low & Antiepileptic & Long & 18 months & \begin{tabular}{|l|} 
Att, CF+ R, \\
Lan., PM
\end{tabular} \\
\hline Werry et al. ${ }^{84}$ & 1975 & New Zealand & $\begin{array}{l}\text { RCT (Crosso- } \\
\text { ver) }\end{array}$ & $21(21 / 0)$ & $8.7(1.7)$ & Incontinence & Imipramine & High & $\begin{array}{l}\begin{array}{l}\text { Antidepres- } \\
\text { sant }\end{array} \\
\end{array}$ & Acute & 3 weeks & Att \\
\hline $\begin{array}{l}\text { Wilson and } \\
\text { Staton }^{85}\end{array}$ & 1984 & USA & $\begin{array}{l}\text { NRCT } \\
\text { (Within) }\end{array}$ & $75(55 / 20)$ & \begin{tabular}{|l}
10.8 \\
$(5.5-16.0)$
\end{tabular} & MDD & $\begin{array}{l}\text { Amitripty- } \\
\text { line, Imipra- } \\
\text { mine }\end{array}$ & High & $\begin{array}{l}\text { Antidepres- } \\
\text { sant }\end{array}$ & Long & $>3$ months & $\begin{array}{l}\text { Att., CF + R, } \\
\text { EF, Int., Lan., } \\
\text { PM }\end{array}$ \\
\hline Yepes et al. ${ }^{86}$ & 1977 & USA & $\begin{array}{l}\text { RCT (Crosso- } \\
\text { ver) }\end{array}$ & $22(21 / 1)$ & $\begin{array}{l}9.2 \\
(7.3-12.3)\end{array}$ & $\begin{array}{l}\text { Hyperactivity/ } \\
\text { aggressive behav- } \\
\text { iour }\end{array}$ & $\begin{array}{l}\text { Amitripty- } \\
\text { line }\end{array}$ & High & $\begin{array}{l}\text { Antidepres- } \\
\text { sant }\end{array}$ & Acute & 2 weeks & Att., EF \\
\hline Yuan et al. ${ }^{87}$ & 2018 & China & $\begin{array}{l}\text { RCT } \\
\text { (Between*) }\end{array}$ & $124(85 / 39)$ & $6.5(2.0)$ & ID & Lithium & Low & \begin{tabular}{|l|}
$\begin{array}{l}\text { Antipsy- } \\
\text { chotic }\end{array}$ \\
\end{tabular} & Long & 3 months & Int \\
\hline
\end{tabular}

Table 1. Demographic, sample, anticholinergic medication and cognitive outcome characteristics for included studies within meta-analysis for cognitive outcomes on and off anticholinergic medication. ${ }^{*}$ Age reported as mean (SD or range) or median [range]. ${ }^{*}$ Sufficient data available for both within- and between-groups design. Selection was made using protocol outlined in "Methods". Studies without description of gender split did not report this information in their original study. $A D H D$ attention deficit hyperactive disorder, Att. attention, $B P D$ bipolar disorder, $C D$ conduct disorder, $C F+R$ concept formation and reasoning, Comp. composite score, $D B D$ disruptive behaviour disorder, $E F$ executive function, $I D$ intellectual disability, Int. intelligence, IQ intelligence quotient, Lan. language, $M D D$ major depressive disorder, Mem. memory, NRCT non-randomized controlled trial, NR not reported, $P D D$ pervasive developmental disorder, Perc. perception, $P M$ psychomotor functioning, $R C T$ randomized controlled trial, $S A R$ seasonal affective rhinitis, TBI traumatic brain injury.

performance also differ between children and adults. Cognitive performance in children is typically reported as test scores on a continuum, while in adults (especially those in late-life), a dichotomous classification of Neurocognitive Disorders is primarily used (e.g. presence versus absence of mild cognitive impairment or dementia). Study designs and differences in classification of cognition therefore may also underlie differences in the patterns of effects observed in children versus older adults, including the finding that anticholinergic antidepressants displayed a positive association with cognition (albeit with a small effect size, which was not significant when only high-quality studies were included). This small positive effect may be due to the short-term nature of the studies included here and is consistent with a meta-analysis of randomised control trials in adult samples ${ }^{92}$. We do not 
Study

Kwon 2013

O'Dougherty 1987

Piccinelli 2010

Erickson 1984

Aldenkamp 1993

de Graaf 2011

Tonnby 1994

Gualtieri 1988

Sommer 2005

Operto 2019

Ferguson 2012

Gualtieri 1991

Werry 1975

Schlieper 1991

Klein 1990

Bender 2004

Donati 2007

Stevenson 2002

Farmer 2017

Chen 2001

Rappaport 1989

Pandina 2009

Yuan 2018

Seidel 1999

Eun $2012 b$

Jung 2015

Beers 2005

de Graaf 2013

Giramonti 2008

Forsythe 1991

Carlson 1992

Tzitiridou 2005

Aman 2009

Platt 1981

Robles 2011

Yepes 1977

Freibergs 1968

Platt 1984

Barrickman 1991

Shehab 2016

Eun 2012a

Gunther 2006

Bender 1991

Aman 2008

Wilson 1984

Troost 2006

Overall effect

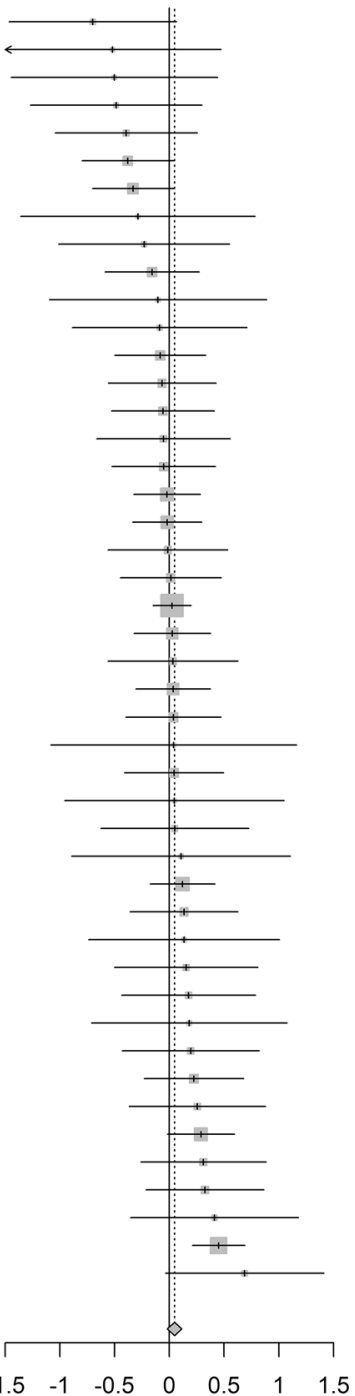

Hedges' g $95 \% \mathrm{Cl}$

$-0.70[-1.46 ; 0.07]$

$-0.52[-1.51 ; 0.47]$

$-0.50[-1.44 ; 0.44]$

$-0.49[-1.27 ; 0.30]$

$-0.39[-1.04 ; 0.25]$

$-0.38 \quad[-0.80 ; 0.04]$

$-0.33[-0.70 ; 0.04]$

$-0.29[-1.36 ; 0.78]$

$-0.23[-1.01 ; 0.55]$

$-0.16[-0.59 ; 0.27]$

-0.10 [-1.10;0.89]

$-0.09[-0.89 ; 0.71]$

$-0.08[-0.50 ; 0.33]$

$-0.07 \quad[-0.56 ; 0.43]$

$-0.06 \quad[-0.53 ; 0.41]$

$-0.05[-0.66 ; 0.56]$

$-0.05[-0.52 ; 0.42]$

$-0.02 \quad[-0.33 ; 0.28]$

$-0.02 \quad[-0.34 ; 0.30]$

$-0.01[-0.56 ; 0.53]$

$0.01[-0.45 ; 0.47]$

$0.02[-0.15 ; 0.20]$

$0.03[-0.32 ; 0.38]$

$0.03 \quad[-0.56 ; 0.63]$

$0.04[-0.30 ; 0.37]$

$0.04[-0.40 ; 0.47]$

$0.04[-1.08 ; 1.16]$

$0.04[-0.41 ; 0.50]$

$0.05[-0.96 ; 1.05]$

$0.05[-0.63 ; 0.72]$

$0.11[-0.89 ; 1.11]$

$0.12[-0.17 ; 0.42]$

$0.13[-0.36 ; 0.63]$

$0.13[-0.74 ; 1.01]$

$0.15[-0.50 ; 0.81]$

$0.18[-0.44 ; 0.79]$

$0.18[-0.71 ; 1.08]$

$0.20[-0.43 ; 0.82]$

$0.22[-0.23 ; 0.68]$

$0.26[-0.37 ; 0.88]$

$0.29[-0.02 ; 0.60]$

$0.31 \quad[-0.26 ; 0.88]$

$0.33[-0.21 ; 0.86]$

$0.41 \quad[-0.35 ; 1.18]$

$0.45[0.21 ; 0.69]$

$0.69[-0.04 ; 1.41]$

$0.05[-0.02 ; 0.11]$

Figure 2. Forest plot for overall cognition analysis.

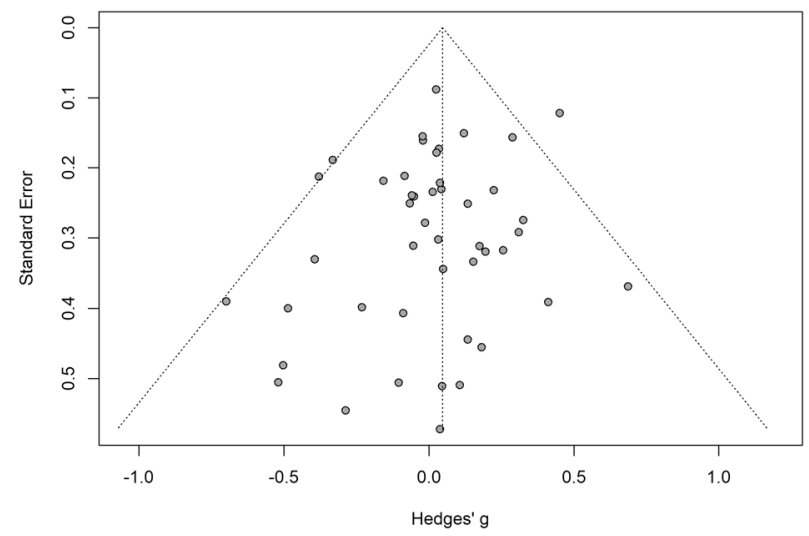

Figure 3. Funnel plot for overall cognition analysis. 


\begin{tabular}{|c|c|c|c|c|c|c|c|c|c|c|}
\hline \multirow[b]{2}{*}{ Subgroup analysis } & \multicolumn{4}{|c|}{ Pooled estimate } & \multicolumn{3}{|c|}{ Heterogeneity } & \multicolumn{3}{|c|}{$\begin{array}{l}\text { Test of between- } \\
\text { subgroups differences }\end{array}$} \\
\hline & $\mathbf{k}$ & g & 95\% CI & p value & $\mathrm{Tau}^{2}$ & $\mathrm{I}^{2}$ & $\mathbf{Q}$ & $\mathbf{Q}$ & df & p value \\
\hline \multicolumn{8}{|l|}{ Drug class } & 9.98 & 5 & 0.08 \\
\hline Antiepileptic & 14 & -0.03 & $-0.17-0.11$ & 0.63 & 0 & 9.67 & 14.39 & & & \\
\hline Antipsychotic & 14 & 0.06 & $-0.03-0.16$ & 0.19 & 0 & 0 & 7.44 & & & \\
\hline Antidepressant & 7 & 0.24 & $0.01-0.47$ & 0.04 & 0 & 13.22 & 6.91 & & & \\
\hline Respiratory & 5 & 0.02 & $-0.15-0.19$ & 0.75 & 0 & 0 & 1.48 & & & \\
\hline Opioid analgesic & 3 & -0.18 & $-0.79-0.44$ & 0.34 & 0 & 0 & 1.84 & & & \\
\hline Urological & 2 & -0.13 & $-1.83-1.58$ & 0.52 & 0 & 0 & 0.18 & & & \\
\hline \multicolumn{8}{|l|}{ Potency } & 0.71 & 1 & 0.40 \\
\hline Low & 36 & 0.02 & $-0.05-0.09$ & 0.50 & 0 & 0 & 27.40 & & & \\
\hline High & 10 & 0.11 & $-0.11-0.33$ & 0.29 & 0.01 & 28.02 & 12.50 & & & \\
\hline \multicolumn{8}{|l|}{ Length of administration } & 2.62 & 2 & 0.27 \\
\hline Current and long-term & 29 & 0.07 & $-0.03-0.17$ & 0.19 & 0.01 & 23.39 & 36.55 & & & \\
\hline Current and acute & 20 & 0.05 & $-0.04-0.14$ & 0.25 & 0 & 0 & 8.06 & & & \\
\hline Historical & 3 & -0.18 & $-0.79-0.44$ & 0.34 & 0.00 & 0 & 1.84 & & & \\
\hline \multicolumn{8}{|l|}{ Cognitive domain } & 5.59 & 7 & 0.59 \\
\hline Attention & 37 & 0.04 & $-0.04-0.12$ & 0.32 & 0 & 0 & 35.49 & & & \\
\hline Psychomotor functioning & 17 & -0.10 & $-0.32-0.11$ & 0.32 & 0.10 & 63.24 & 43.52 & & & \\
\hline Concept formation and reasoning & 13 & 0.14 & $-0.02-0.30$ & 0.08 & 0.01 & 15.96 & 14.28 & & & \\
\hline Perception & 3 & 0.25 & $-0.90-1.39$ & 0.45 & 0.11 & 50.18 & 4.01 & & & \\
\hline Memory & 16 & 0.04 & $-0.06-0.14$ & 0.40 & 0 & 0 & 9.05 & & & \\
\hline Executive function & 15 & -0.01 & $-0.27-0.24$ & 0.91 & 0.12 & 48.50 & 27.19 & & & \\
\hline Intelligence & 14 & 0.08 & $-0.18-0.33$ & 0.53 & 0.13 & 76.23 & 54.70 & & & \\
\hline Language & 6 & 0.11 & $-0.07-0.29$ & 0.17 & 0 & 0 & 4.54 & & & \\
\hline
\end{tabular}

Table 2. Pooled estimates for subgroup analyses by anticholinergic drug class, potency, length of administration and cognitive domains.

know the effects of the long-term use anticholinergic antidepressants in children. Notably, a small positive effect of anticholinergic medication on memory was found when only including studies of high quality. Whether this is a true effect, which is counter to that found in adults ${ }^{93}$, needs to be replicated in future studies. Lastly, there are important biological differences between children and adults that would modify the psychopharmacological effects of anticholinergic medications, particularly blood brain permeability ${ }^{94,95}$.

This study is not without limitations. The included studies were biased in terms of geographical representativeness. Fourteen studies were excluded at the full-text stage as they were not in English (of 323) and we do not know if any would have met inclusion criteria; although, given the low number, they would unlikely have changed the conclusions. Authors of papers were contacted, but we either had no response or the author was unable to provide us with the necessary data where it was not presented in text. We assessed the effect of duration of exposure on cognitive outcomes, when total dose or volume of exposure may have been more appropriate. However, this information was inconsistently reported or not reported at all in many of the studies. Therefore, duration of use was used as the best proxy for volume of exposure, with the assumption that longer duration of use would equate to higher volume of exposure. Only 21 of the 100 high- or low-potency anticholinergics identified in a systematic review of anticholinergic medications by Duran et al. ${ }^{12}$ were used in the studies included in this meta-analysis. It may be that different results would be seen had children been exposed to a wider range of anticholinergic medicines. Positively, the vast majority of studies (all but two) utilised valid and reliable cognitive outcome measures, as catalogued specifically or adapted from those detailed in Lezak et al. ${ }^{96}$.

\section{Conclusion}

By pooling effects across previous literature, anticholinergic medications do not appear to detrimentally affect cognitive function in children. In fact, there may be a small positive cognitive benefit of anticholinergic antidepressants, at least in the short-term. Our findings appear to conflict with reviews in older adults, and future studies will have to disentangle the reasons for this. 
Study

Antiepileptic Kwon 2013

O'Dougherty 1987 Piccinelli 2010 Aldenkamp 1993

Tonnby 1994 Operto 2019 Donati 2007

Chen 2001 Seidel 1999 Eun 2012b Jung 2015

Forsythe 1991 Tzitiridou 2005 Eun 2012a

Overall effect

Antipsychotic Erickson 1984

Klein 1990

Farmer 2017

Pandina 2009

Yuan 2018

Carlson 1992

Aman 2009

Platt 1981

Robles 2011

Freibergs 1968

Platt 1984

Gunther 2006

Aman 2008

Troost 2006

Overall effect

Antidepressant

Gualtieri 1988

Gualtieri 1991

Werry 1975

Yepes 1977

Barrickman 1991

Shehab 2016

Wilson 1984

Overall effect

Respiratory

Schlieper 1991

Bender 2004

Stevenson 2002

Rappaport 1989

Bender 1991

Overall effect

Opioid analgesic de Graaf 2011

Ferguson 2012

de Graaf 2013

Overall effect

Urological

Sommer 2005

Giramonti 2008

Overall effect

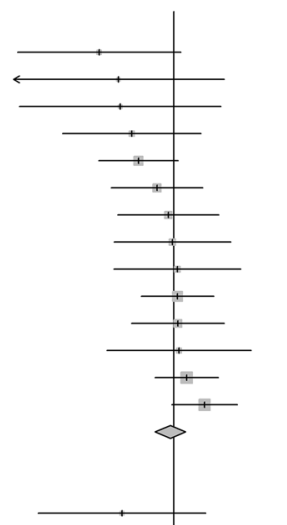

Hedges' 9

$95 \% \mathrm{Cl}$

$-0.70[-1.46 ; 0.07]$

$-0.52[-1.51 ; 0.47]$

$-0.50[-1.44 ; 0.44]$

$-0.39[-1.04 ; 0.25]$

$-0.33[-0.70 ; 0.04]$

$-0.16[-0.59 ; 0.27]$

$-0.05[-0.52 ; 0.42]$

$-0.01[-0.56 ; 0.53]$

$0.03[-0.56 ; 0.63]$

$0.04[-0.30 ; 0.37]$

$0.04[-0.40 ; 0.47]$

$0.05[-0.63 ; 0.72]$

$0.12[-0.17 ; 0.42]$

$0.29[-0.02 ; 0.60]$

$-0.03[-0.17 ; 0.11]$

$-0.49 \quad[-1.27 ; 0.30]$

$-0.06[-0.53 ; 0.41]$

$-0.02[-0.34 ; 0.30]$

$0.02[-0.15 ; 0.20]$

$0.03[-0.32 ; 0.38]$

$0.11[-0.89 ; 1.11]$

$0.13[-0.36 ; 0.63]$

$0.13[-0.74 ; 1.01]$

$0.15[-0.50 ; 0.81]$

$0.18[-0.71 ; 1.08]$

$0.20[-0.43 ; 0.82]$

$0.31 \quad[-0.26 ; 0.88]$

$0.41[-0.35 ; 1.18]$

$0.69[-0.04 ; 1.41]$

$0.06[-0.03 ; 0.16]$

$-0.29 \quad[-1.36 ; 0.78]$

$-0.09[-0.89 ; 0.71]$

$-0.08[-0.50 ; 0.33]$

$0.18[-0.44 ; 0.79]$

$0.22[-0.23 ; 0.68]$

$0.26[-0.37 ; 0.88]$

$0.45[0.21 ; 0.69]$

$0.24[0.01 ; 0.47]$

$-0.07 \quad[-0.56 ; 0.43]$

$-0.05[-0.66 ; 0.56]$

$-0.02[-0.33 ; 0.28]$

$0.01[-0.45 ; 0.47]$

$0.33[-0.21 ; 0.86]$

$0.02[-0.15 ; 0.19]$

$-0.38[-0.80 ; 0.04]$

$-0.10[-1.10 ; 0.89]$

$0.04[-0.41 ; 0.50]$

$-0.18[-0.79 ; 0.44]$

$-0.23[-1.01 ; 0.55]$

$0.05[-0.96 ; 1.05]$

$-0.13[-1.83 ; 1.58]$

Figure 4. Forest plot for medication class sub-analysis. 
Study

Low

Kwon 2013

O'Dougherty 1987

Piccinelli 2010

Aldenkamp 1993

de Graaf 2011

Tonnby 1994

Operto 2019

Ferguson 2012

Schlieper 1991

Bender 2004

Donati 2007

Stevenson 2002

Farmer 2017

Chen 2001

Rappaport 1989

Pandina 2009

Yuan 2018

Seidel 1999

Eun 2012b

Jung 2015

Beers 2005

de Graaf 2013

Forsythe 1991

Carlson 1992

Tzitiridou 2005

Aman 2009

Platt 1981

Robles 2011

Platt 1984

Barrickman 1991

Shehab 2016

Eun 2012a

Gunther 2006

Bender 1991

Aman 2008

Troost 2006

Overall effect

High

Erickson 1984

Gualtieri 1988

Sommer 2005

Gualtieri 1991

Werry 1975

Klein 1990

Giramonti 2008

Yepes 1977

Freibergs 1968

Wilson 1984

Overall effect

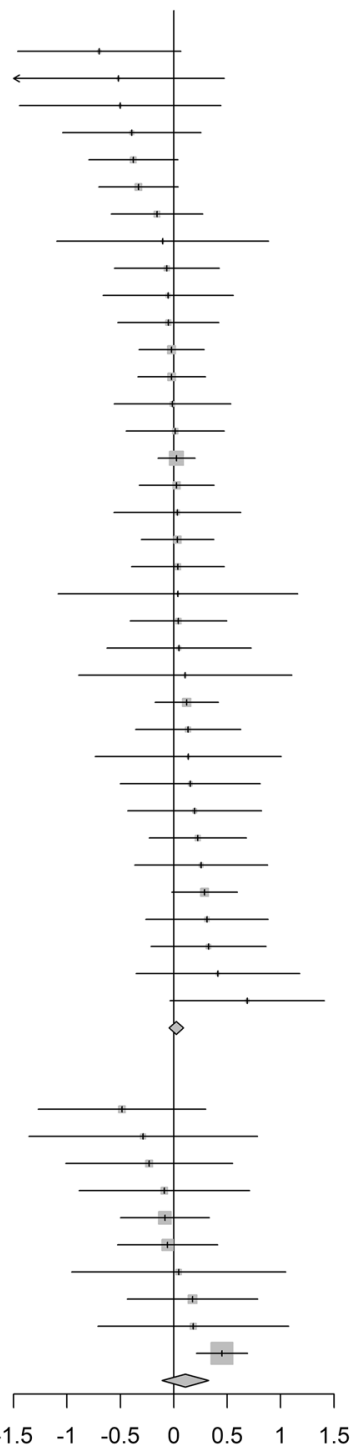

Hedges' $g$

$95 \% \mathrm{Cl}$

$-0.70[-1.46 ; 0.07]$

$-0.52[-1.51 ; 0.47]$

$-0.50[-1.44 ; 0.44]$

$-0.39[-1.04 ; 0.25]$

$-0.38[-0.80 ; 0.04]$

$-0.33[-0.70 ; 0.04]$

$-0.16[-0.59 ; 0.27]$

$-0.10[-1.10 ; 0.89]$

$-0.07 \quad[-0.56 ; 0.43]$

$-0.05[-0.66 ; 0.56]$

$-0.05 \quad[-0.52 ; 0.42]$

$-0.02[-0.33 ; 0.28]$

$-0.02[-0.34 ; 0.30]$

$-0.01[-0.56 ; 0.53]$

$0.01[-0.45 ; 0.47]$

$0.02[-0.15 ; 0.20]$

$0.03[-0.32 ; 0.38]$

$0.03[-0.56 ; 0.63]$

$0.04[-0.30 ; 0.37]$

$0.04[-0.40 ; 0.47]$

$0.04[-1.08 ; 1.16]$

$0.04[-0.41 ; 0.50]$

$0.05[-0.63 ; 0.72]$

$0.11[-0.89 ; 1.11]$

$0.12[-0.17 ; 0.42]$

$0.13[-0.36 ; 0.63]$

$0.13[-0.74 ; 1.01]$

$0.15[-0.50 ; 0.81]$

$0.20[-0.43 ; 0.82]$

$0.22[-0.23 ; 0.68]$

$0.26 \quad[-0.37 ; 0.88]$

$0.29[-0.02 ; 0.60]$

$0.31 \quad[-0.26 ; 0.88]$

$0.33[-0.21 ; 0.86]$

$0.41[-0.35 ; 1.18]$

$0.69[-0.04 ; 1.41]$

$0.02[-0.05 ; 0.09]$

$-0.49[-1.27 ; 0.30]$

$-0.29[-1.36 ; 0.78]$

$-0.23[-1.01 ; 0.55]$

$-0.09[-0.89 ; 0.71]$

$-0.08[-0.50 ; 0.33]$

$-0.06 \quad[-0.53 ; 0.41]$

$0.05[-0.96 ; 1.05]$

$0.18[-0.44 ; 0.79]$

$0.18[-0.71 ; 1.08]$

$0.45[0.21 ; 0.69]$

$0.11[-0.11 ; 0.33]$

Figure 5. Forest plot for anticholinergic potency sub-analysis. 
Study

Current + long-term Kwon 2013

O'Dougherty 1987 Piccinelli 2010

Erickson 1984

Aldenkamp 1993

Tonnby 1994

Operto 2019

Donati 2007

Stevenson 2002

Klein 1990

Chen 2001

Forsythe 1991

Pandina 2009

Yuan 2018

Seidel 1999

Eun 2012b

Jung 2015

Beers 2005

Tzitiridou 2005

Robles 2011

Freibergs 1968

Barrickman 1991

Shehab 2016

Eun 2012a

Aman 2008

Carlson 1992

Wilson 1984

Bender 1991

Troost 2006

Overall effect

Current + acute

Gualtieri 1988

Sommer 2005

Carlson 1992

Klein 1990

Gualtieri 1991

Werry 1975

Schlieper 1991

Bender 2004

Farmer 2017

Rappaport 1989

Giramonti 2008

Bender 1991

Forsythe 1991

Aman 2009

Platt 1981

Yepes 1977

Platt 1984

Gunther 2006

Aman 2008

Troost 2006

Overall effect

Historical

de Graaf 2011

Ferguson 2012

de Graaf 2013

Overall effect

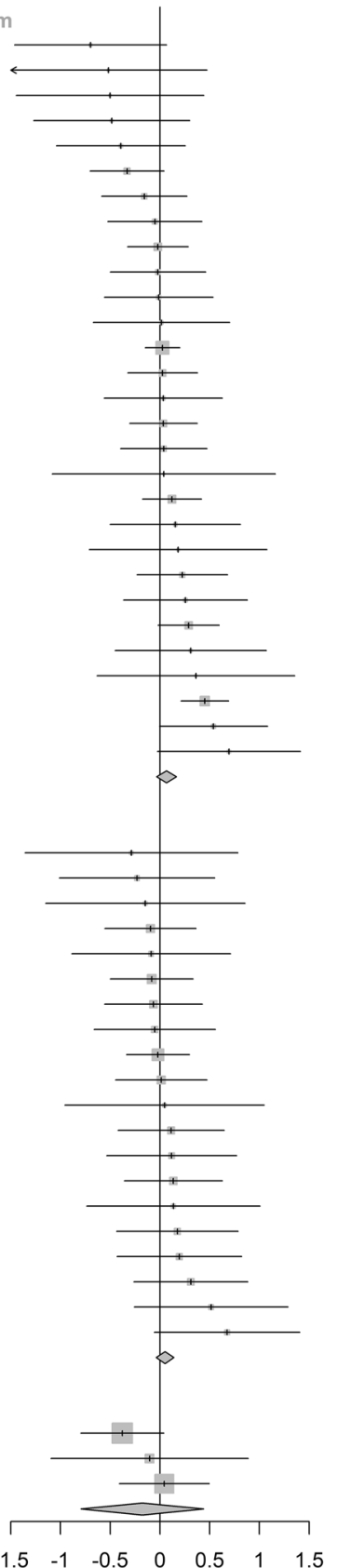

Hedges' g

$95 \% \mathrm{CI}$

$-0.70[-1.46 ; 0.07]$

$-0.52[-1.51 ; 0.47]$

$-0.50[-1.44 ; 0.44]$

$-0.49[-1.27 ; 0.30]$

$-0.39[-1.04 ; 0.25]$

$-0.33[-0.70 ; 0.04]$

$-0.16[-0.59 ; 0.27]$

$-0.05 \quad[-0.52 ; 0.42]$

$-0.02[-0.33 ; 0.28]$

$-0.02[-0.50 ; 0.46]$

$-0.01 \quad[-0.56 ; 0.53]$

$0.02[-0.67 ; 0.70]$

$0.02[-0.15 ; 0.20]$

$0.03[-0.32 ; 0.38]$

$0.03 \quad[-0.56 ; 0.63]$

$0.04[-0.30 ; 0.37]$

$0.04[-0.40 ; 0.47]$

$0.04[-1.08 ; 1.16]$

$0.12[-0.17 ; 0.42]$

$0.15[-0.50 ; 0.81]$

$0.18[-0.71 ; 1.08]$

$0.22[-0.23 ; 0.68]$

$0.26[-0.37 ; 0.88]$

$0.29[-0.02 ; 0.60]$

$0.31[-0.45 ; 1.07]$

$0.36[-0.63 ; 1.36]$

$0.45[0.21 ; 0.69]$

$0.54[0.00 ; 1.08]$

$0.69[-0.02 ; 1.41]$

$0.07[-0.03 ; 0.17]$

$-0.29[-1.36 ; 0.78]$

$-0.23[-1.01 ; 0.55]$

$-0.15[-1.15 ; 0.86]$

$-0.10 \quad[-0.55 ; 0.36]$

$-0.09[-0.89 ; 0.71]$

$-0.08[-0.50 ; 0.33]$

$-0.07[-0.56 ; 0.43]$

$-0.05[-0.66 ; 0.56]$

$-0.02[-0.34 ; 0.30]$

$0.01[-0.45 ; 0.47]$

$0.05[-0.96 ; 1.05]$

$0.11 \quad[-0.42 ; 0.65]$

$0.12[-0.54 ; 0.77]$

$0.13[-0.36 ; 0.63]$

$0.13[-0.74 ; 1.01]$

$0.18[-0.44 ; 0.79]$

$0.20[-0.43 ; 0.82]$

$0.31[-0.26 ; 0.88]$

$0.51[-0.26 ; 1.29]$

$0.67[-0.06 ; 1.41]$

$0.05[-0.04 ; 0.14]$

$-0.38[-0.80 ; 0.04]$

$-0.10[-1.10 ; 0.89]$

$0.04[-0.41 ; 0.50]$

$-0.18[-0.79 ; 0.44]$

Figure 6. Forest plot for length of administration sub-analysis. 
Study

Attention

Erickson 1984

Tonnby 1994

Kwon 2013

Aldenkamp 1993

Ferguson 2012

O'Dougherty 1987

Gualtieri 1991

Seidel 1999

Bender 2004

Schlieper 1991

de Graaf 2013

Werry 1975

Sommer 2005

Klein 1990

Donati 2007

Tzitiridou 2005

Beers 2005

Farmer 2017

Pandina 2009

Gualtieri 1988

Barrickman 1991

Forsythe 1991

Aman 2009

Rappaport 1989

Robles 2011

Eun 2012b

Giramonti 2008

Yepes 1977

Carlson 1992

Shehab 2016

Aman 2008

Platt 1981

Gunther 2006

Bender 1991

Platt 1984

Wilson 1984

Troost 2006

Overall effect

Psychomotor Functioning

O'Dougherty 1987

Gualtieri 1991

Gualtieri 1988

Aldenkamp 1993

de Graaf 2011

Tonnby 1994

de Graaf 2013

Klein 1990

Seidel 1999

Rappaport 1989

Donati 2007

Eun 2012b

Aman 2008

Robles 2011

Tzitiridou 2005

Aman 2009

Wilson 1984

Overall effect

Concept Formation \& Reasonin

Piccinelli 2010

Aman 2008

Kwon 2013

Beers 2005

Ferguson 2012

Eun 2012

Klein 1990

Tzitiridou 2005

Robles 2011

Seidel 1999

Freibergs 1968

de Graaf 2013

Wilson 1984

Overall effect

Perception

Donati 2007

Robles 2011

Aman 2008

Overall effect

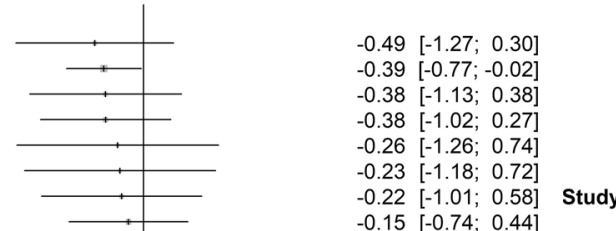

$-0.15[-0.74 ; 0.44]$

$-0.13[-0.74 ; 0.48]$

$-0.09[-0.55 ; 0.36]$ Sommer 2005

$-0.08 \quad[-0.50 ; 0.33] \quad$ Carlson 1992

$-0.08[-0.86$

$-0.08[-0.55 ; 0.40]$

Carlson 1992

O'Dougherty 1987

Klein 1990

$-0.07[-0.54 ; 0.40]$ Giramonti 2008

$-0.05[-0.38 ; 0.28]$ Donati 2007

$-0.04[-1.16 ; 1.07]$

-0.02 [-0.3.

$-0.01[-0.19 ; 0.30]$

Donati 2007

Pandina 2009

Forsythe 1991

$-0.00[-1.15 ; 1.15] \quad$ Rappaport 1989

$-0.00[-0.45 ; 0.45] \quad$ Bender 2004

$0.00[-0.68 ; 0.69] \quad$ Gualtieri 1991

$0.04[-0.45 ; 0.52]$ Robles 2011

$0.04[-0.42 ; 0.49]$ Seidel 1999

$0.05[-0.63 ; 0.73] \quad$ Aman 2008

$0.11[-0.22 ; 0.44] \quad$ Overall effect

$0.12[-0.88 ; 1.11]$

0.20

Executive Function

$0.22[-0.40 ; 0.84] \quad$ Klein 1990

$0.24[-0.51 ; 0.98] \quad$ Rappaport 1989

$0.25[-0.64 ; 1.14] \quad$ Schlieper 1991

$0.31[-0.27 ; 0.88] \quad$ Platt 1984

$0.33[-0.21 ; 0.86] \quad$ Platt 1981

$0.39[-0.24 ; 1.02]$ Yepes 1977

0.50 [0.26; 0.75] Robles 2011

$0.69[-0.04 ; 1.41]$ de Graaf 2013

$0.04[-0.04 ; 0.12]$ Barrickman 1991

Wilson 1984

Beers 2005

-1.30 [-2 39; -0.20] Shehab 2016

$-1.04[-1.8$

$-0.85[-1.76 ; 0.05]$ Carlson 1992

$-0.47[-1.12 ; 0.18] \quad$ Overall effect

$-0.34[-0.76 ; 0.07]$

-0.31 [-0.68;0.06] Intelligence

$-0.25[-0.69 ; 0.19] \quad K w o n 2013$

$-0.13[-0.60 ; 0.34]$ Piccinelli 2010

$-0.08[-0.66 ; 0.50]$ de Graaf 2011

$-0.06[-0.52 ; 0.39] \quad$ Ferguson 2012

$-0.05[-0.52 ; 0.41]$

$-0.03[-0.36 ; 0.30]$

$0.15[-0.48 ; 0.78]$

$0.19[-0.49 ; 0.88]$

de Graaf 2013

Klein 1990

Chen 2001

Yuan 2018

Jung 2015

0.43 [-0.07; 0.93] Eun 2012b

$0.45[0.21 ; 0.69] \quad$ Tzitiridou 2005

$0.45[0.21 ; 0.69]$
$-0.10[-0.32 ; 0.11]$

$-0.54[-1.48 ; 0.41] \quad$ Seidel 1999 Eun 2012a Barrickman 199 Wilson 1984

$-0.37[-1.00 ; 0.26] \quad$ Overall effect

$-0.28[-0.99 ; 0.44]$

$-0.28[-1.38 ; 0.83]$

$0.05[-0.28 ; 0.38]$

$0.07[-0.38 ; 0.52]$

$0.08[-0.21 ; 0.37]$

$0.13[-0.55 ; 0.81]$

$0.18[-0.40 ; 0.75]$

$0.18[-0.71 ; 1.08]$

$0.42[-0.04 ; 0.87]$

$0.43[0.19 ; 0.66]$

$0.14[-0.02 ; 0.30]$

Eun 2012b

Klein 1990

zitiridou 2005

Wilson 1984

Ferguson 2012

Overall effect

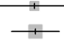

$\infty$

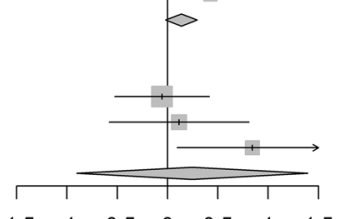

$\begin{array}{lllllll}-1.5 & -1 & -0.5 & 0 & 0.5 & 1 & 1.5\end{array}$

$-0.05[-0.52 ; 0.42]$

$0.11[-0.58 ; 0.81]$

$0.84[0.10 ; 1.59]$

$0.25[-0.90 ; 1.39]$
Hedges' g

$95 \% \mathrm{Cl}$

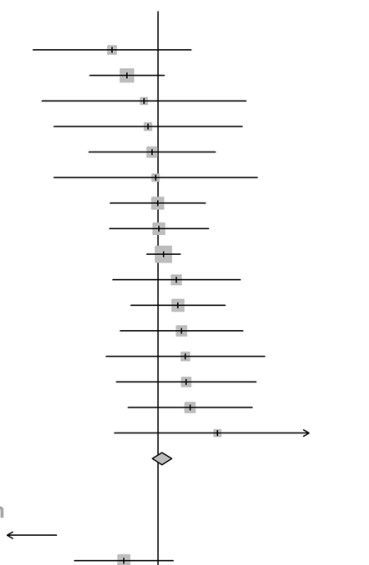

$-0.46[-1.24 ; 0.33]$

$0.31[-0.68 ; 0.06]$

$-0.14[-1.15 ; 0.87]$

$-0.10[-1.03 ; 0.84]$

$-0.06[-0.69 ; 0.57]$

$-0.02[-1.03 ; 0.99]$

$-0.00[-0.48 ; 0.47]$

$0.01[-0.48 ; 0.50]$

$0.05[-0.11 ; 0.22]$

$0.18[-0.45 ; 0.82]$

$0.20[-0.27 ; 0.67]$

$0.23[-0.38 ; 0.84]$

$0.27[-0.52 ; 1.06]$

$0.28[-0.41 ; 0.97]$

$0.32[-0.30 ; 0.94]$

$0.59[-0.43 ; 1.61]$

$0.04[-0.06 ; 0.14]$

$-1.87[-2.73 ;-1.01]$

$-0.34[-0.83 ; 0.15]$

$-0.12[-0.58 ; 0.35]$

$-0.10[-0.59 ; 0.39]$

$-0.04[-0.66 ; 0.58]$

$0.02[-0.83 ; 0.87]$

$0.03[-0.64 ; 0.71]$

$0.08[-0.65 ; 0.81]$

$0.10[-0.35 ; 0.56]$

$0.18[-0.26 ; 0.61]$

0.23 [ $0.00 ; 0.45$ ]

$0.25[-0.89 ; 1.38]$

$0.27[-0.36 ; 0.89]$

$0.33[-0.25 ; 0.90]$

0.40 [-0.59; 1.39$]$

$-0.01[-0.27 ; 0.24]$

$-0.91[-1.66 ;-0.16]$ -0.47 [-1.41; 0.47$]$ $-0.42[-0.83 ; 0.00]$ $-0.24[-1.23 ; 0.74]$

$-0.11[-0.54 ; 0.33]$

$-0.06[-0.51 ; 0.39]$

-0.01 [-0.56; 0.53$]$

0.03 [-0.32;0.38]

$0.04[-0.40 ; 0.47]$

$0.09[-0.24 ; 0.42]$

0.28 [0.05; 0.52$]$

0.29 [-0.02; 0.60$]$

$0.72[0.23 ; 1.21]$

0.86 [0.60; 1.12]

$0.08[-0.18 ; 0.33]$

$-0.22[-0.80 ; 0.35]$

$-0.06[-0.44 ; 0.32]$

$-0.03[-0.48 ; 0.42]$

$0.09[-0.19 ; 0.37]$

$0.27[0.04 ; 0.50]$

$0.30[-0.69 ; 1.28]$

$0.11[-0.07 ; 0.29]$

Figure 7. Forest plot for cognitive domain sub-analysis. 


\section{Data availability}

All data and code available at https://github.com/ericaghezzi/anticholinergic_med_metaanalysis.

Received: 23 September 2020; Accepted: 14 December 2020

Published online: 08 January 2021

\section{References}

1. Cebron Lipovec, N., Jazbar, J. \& Kos, M. Anticholinergic burden in children, adults and older adults in Slovenia: A Nationwide database study. Sci. Rep. 10(1), 9337 (2020).

2. Santos, J. D., Lopes, R. I. \& Koyle, M. A. Bladder and bowel dysfunction in children: An update on the diagnosis and treatment of a common, but underdiagnosed pediatric problem. Can. Urol. Assoc. J. 11(1-2 Suppl), S64-S72 (2017).

3. Rhee, T. G., Choi, Y. C., Ouellet, G. M. \& Ross, J. S. National prescribing trends for high-risk anticholinergic medications in older adults. J. Am. Geriatr. Soc. 66(7), 1382-1387 (2018).

4. Richardson, K. et al. Anticholinergic drugs and risk of dementia: Case-control study. BMJ 361, k1315 (2018).

5. Campbell, N. L., Lane, K. A., Gao, S., Boustani, M. A. \& Unverzagt, F. Anticholinergics influence transition from normal cognition to mild cognitive impairment in older adults in primary care. Pharmacotherapy 38(5), 511-519 (2018).

6. Coupland, C. A. C. et al. Anticholinergic drug exposure and the risk of dementia: A nested case-control study. JAMA Intern. Med. 179(8), 1084-1093 (2019).

7. Fox, C. et al. Effect of medications with anti-cholinergic properties on cognitive function, delirium, physical function and mortality: A systematic review. Age Ageing 43(5), 604-615 (2014).

8. Ruxton, K., Woodman, R. J. \& Mangoni, A. A. Drugs with anticholinergic effects and cognitive impairment, falls and all-cause mortality in older adults: A systematic review and meta-analysis. Br. J. Clin. Pharmacol. 80(2), 209-220 (2015).

9. Pieper, N. T. et al. Anticholinergic drugs and incident dementia, mild cognitive impairment and cognitive decline: A meta-analysis. Age Ageing 49, 939-947 (2020).

10. Australian Centre for Asthma Monitoring: Asthma in Australian children: findings from Growing Up in Australia, the Longitudinal Study of Australian Children. Cat. No. ACM 17. AIHW, Canberra (2009).

11. Caldwell, P., Edgar, D., Hodson, E. \& Craig, J. Bedwetting and toileting problems in children. MJA 182(4), 190-195 (2005).

12. Durán, C. E., Azermai, M. \& Vander Stichele, R. H. Systematic review of anticholinergic risk scales in older adults. Eur. J. Clin. Pharmacol. 69(7), 1485-1496 (2013).

13. Gray, S. L. et al. Cumulative use of strong anticholinergics and incident dementia: A prospective cohort study. JAMA Intern. Med. 175(3), 401-407 (2015).

14. McKeith, I. et al. Efficacy of rivastigmine in dementia with Lewy bodies: A randomised, double-blind, placebo-controlled international study. Lancet 356(9247), 2031-2036 (2000).

15. Klinkenberg, I., Sambeth, A. \& Blokland, A. Acetylcholine and attention. Behav. Brain Res. 221(2), 430-442 (2011).

16. Parikh, V. \& Sarter, M. Cholinergic mediation of attention: Contributions of phasic and tonic increases in prefrontal cholinergic activity. Ann. N. Y. Acad. Sci. 1129, 225-235 (2008).

17. Gill, T. M., Sarter, M. \& Givens, B. Sustained visual attention performance-associated prefrontal neuronal activity: Evidence for cholinergic modulation. J. Neurosci. 20(12), 4745-4757 (2000).

18. Moher, D., Liberati, A., Tetzlaff, J. \& Altman, D. G. Preferred reporting items for systematic reviews and meta-analyses: The PRISMA statement. BMJ 339, b2535 (2009).

19. Shamseer, L. et al. Preferred reporting items for systematic review and meta-analysis protocols (PRISMA-P) 2015: Elaboration and explanation. BMJ 349, g7647 (2015).

20. Tufanaru C, Munn Z, Aromataris E, Campbell J, Hopp L: Chapter 3: Systematic reviews of effectiveness. In: Joanna Briggs Institute Reviewer's Manual. edn. Edited by Aromataris E, Munn Z: The Joanna Briggs Institute; 2017.

21. Ma, L.-L. et al. Methodological quality (risk of bias) assessment tools for primary and secondary medical studies: What are they and which is better?. Milit. Med. Res. 7(1), 7 (2020).

22. Sakiris, N. \& Berle, D. A systematic review and meta-analysis of the Unified Protocol as a transdiagnostic emotion regulation based intervention. Clin. Psychol. Rev. 72, 101751 (2019).

23. Ashdown-Franks, G. et al. Is it possible for people with severe mental illness to sit less and move more? A systematic review of interventions to increase physical activity or reduce sedentary behaviour. Schizophr. Res. 202, 3-16 (2018).

24. Ge, S., Zhu, Z., Wu, B. \& McConnell, E. S. Technology-based cognitive training and rehabilitation interventions for individuals with mild cognitive impairment: a systematic review. BMC Geriatr. 18(1), 213 (2018).

25. Hedges, L. V. \& Olkin, I. Statistical Methods for Meta-Analysis (Academic Press, Cambridge, 2014).

26. Schwarzer G: Meta. CRAN 2020: Version 4.13-10.

27. DerSimonian, R. \& Laird, N. Meta-analysis in clinical trials. Control. Clin. Trials 7(3), 177-188 (1986).

28. Cornell, J. E. et al. Random-effects meta-analysis of inconsistent effects: A time for change. Ann. Intern. Med. 160(4), 267-270 (2014).

29. Novianti, P. W., Roes, K. C. \& van der Tweel, I. Estimation of between-trial variance in sequential meta-analyses: A simulation study. Contemp. Clin. Trials 37(1), 129-138 (2014).

30. Veroniki, A. A. et al. Methods to estimate the between-study variance and its uncertainty in meta-analysis. Res. Synth. Methods 7(1), 55-79 (2016).

31. Paule, R. C. \& Mandel, J. Consensus values and weighting factors. J. Res. Natl. Bureau Standards 87(5), 377-385 (1982).

32. Panityakul, T., Bumrungsup, C. \& Knapp, G. On estimating residual heterogeneity in random-effects meta-regression: A comparative study. J. Stat. Theory Appl. 12(3), 253-265 (2013).

33. Hartung, J. \& Knapp, G. On tests of the overall treatment effect in meta-analysis with normally distributed responses. Stat. Med. 20(12), 1771-1782 (2001).

34. Hartung, J. \& Knapp, G. A refined method for the meta-analysis of controlled clinical trials with binary outcome. Stat. Med. 20(24), 3875-3889 (2001).

35. Higgins, J. P., Thompson, S. G., Deeks, J. J. \& Altman, D. G. Measuring inconsistency in meta-analyses. BMJ 327(7414), 557-560 (2003).

36. Lezak MD, Howieson DB, Loring DW, Fischer JS: Neuropsychological assessment: Oxford University Press, USA; 2004.

37. Beers, S. R., Skold, A., Dixon, C. E. \& Adelson, P. D. Neurobehavioral effects of amantadine after pediatric traumatic brain injury: A preliminary report. J. Head Trauma Rehabil. 20(5), 450-463 (2005).

38. Higgins, J. P. T. \& Thompson, S. G. Controlling the risk of spurious findings from meta-regression. Stat. Med. 23(11), 1663-1682 (2004).

39. Sterne, J. A. et al. Recommendations for examining and interpreting funnel plot asymmetry in meta-analyses of randomised controlled trials. BMJ 343, 1 (2011).

40. Egger, M., Smith, G. D., Schneider, M. \& Minder, C. Bias in meta-analysis detected by a simple, graphical test. BMJ 315(7109), 629-634 (1997). 
41. Duval, S. \& Tweedie, R. Trim and fill: a simple funnel-plot-based method of testing and adjusting for publication bias in metaanalysis. Biometrics 56(2), 455-463 (2000).

42. United Nations Department of Economic and Social Affairs. World Economic Situation and Prospects. (2020).

43. Aldenkamp, A. et al. Withdrawal of antiepileptic medication in children-effects on cognitive function: The Multicenter Holmfrid Study. Neurology 43(1), 41 (1993).

44. Aman, M. G. et al. Cognitive effects of risperidone in children with autism and irritable behavior. J. Child Adolesc. Psychopharmacol. 18(3), 227-236 (2008).

45. Aman, M. G. et al. Effects of risperidone on cognitive-motor performance and motor movements in chronically medicated children. Res. Dev. Disabil. 30(2), 386-396 (2009).

46. Barrickman, L., Noyes, R., Kuperman, S., Schumacher, E. \& Verda, M. Treatment of ADHD with fluoxetine: A preliminary trial. J. Am. Acad. Child Adolesc. Psychiatry 30(5), 762-767 (1991).

47. Bender, B. G. \& Milgrom, H. Comparison of the effects of fluticasone propionate aqueous nasal spray and loratadine on daytime alertness and performance in children with seasonal allergic rhinitis. Ann. Allergy Asthma Immunol. 92(3), 344-349 (2004).

48. Bender, B. G., Lerner, J. A., Ikle, D., Comer, C. \& Szefler, S. Psychological change associated with theophylline treatment of asthmatic children: A 6-month study. Pediatr. Pulmonol. 11(3), 233-242 (1991).

49. Carlson, G. A., Rapport, M. D., Pataki, C. S. \& Kelly, K. L. Lithium in hospitalized children at 4 and 8 weeks: Mood, behavior and cognitive effects. J. Child Psychol. Psychiatry 33(2), 411-425 (1992).

50. Chen, Y.-J., Chow, J. C. \& Lee, I.-C. Comparison the cognitive effect of anti-epileptic drugs in seizure-free children with epilepsy before and after drug withdrawal. Epilepsy Res. 44(1), 65-70 (2001).

51. De Graaf, J. et al. Long-term effects of routine morphine infusion in mechanically ventilated neonates on children's functioning: Five-year follow-up of a randomized controlled trial. Pain 152(6), 1391-1397 (2011).

52. de Graaf, J. et al. Does neonatal morphine use affect neuropsychological outcomes at 8 to 9years of age?. Pain 154(3), 449-458 (2013).

53. Donati, F. et al. The cognitive effects of oxcarbazepine versus carbamazepine or valproate in newly diagnosed children with partial seizures. Seizure 16(8), 670-679 (2007).

54. Erickson, W., Yellin, A., Hopwood, J., Realmuto, G., Greenberg, L. The effects of neuroleptics on attention in adolescent schizophrenics. Biol. Psychiatry (1984).

55. Eun, S.-H. et al. Effects of lamotrigine on cognition and behavior compared to carbamazepine as monotherapy for children with partial epilepsy. Brain Develop. 34(10), 818-823 (2012).

56. Eun, S.-H. et al. A multicenter trial of oxcarbazepine oral suspension monotherapy in children newly diagnosed with partial seizures: A clinical and cognitive evaluation. Seizure 21(9), 679-684 (2012).

57. Farmer, C. A. et al. Risperidone added to psychostimulant in children with severe aggression and attention-deficit/hyperactivity disorder: Lack of effect on attention and short-term memory. J. Child Adolesc. Psychopharmacol. 27(2), 117-124 (2017).

58. Ferguson, S. A., Ward, W. L., Paule, M. G., Hall, R. W. \& Anand, K. J. S. A pilot study of preemptive morphine analgesia in preterm neonates: Effects on head circumference, social behavior, and response latencies in early childhood. Neurotoxicol. Teratol. 34(1), 47-55 (2012).

59. Forsythe, I., Butler, R., Berg, I. \& McGuire, R. Cognitive impairment in new cases of epilepsy randomly assigned to carbamazepine, phenytoin and sodium valproate. Dev. Med. Child Neurol. 33(6), 524-534 (1991).

60. Freibergs, V., Douglas, V. I. \& Weiss, G. The effect of chlorpromazine on concept learning in hyperactive children under two conditions of reinforcement. Psychopharmacologia 13(4), 299-310 (1968).

61. Giramonti, K. M., Kogan, B. A. \& Halpern, L. F. The effects of anticholinergic drugs on attention span and short-term memory skills in children. Neurourol. Urodyn. 27(4), 315-318 (2008).

62. Gualtieri, C. T. \& Evans, R. W. Motor performance in hyperactive children treated with imipramine. Percept. Mot. Skills 66(3), 763-769 (1988).

63. Gualtieri, C. T., Keenan, P., Chandler, M. Clinical and neuropsychological effects of desipramine in children with attention deficit hyperactivity disorder. J. Clin. Psychopharmacol. (1991).

64. Günther, T., Herpertz-Dahlmann, B., Jolles, J. \& Konrad, K. The influence of risperidone on attentional functions in children and adolescents with attention-deficit/hyperactivity disorder and co-morbid disruptive behavior disorder. J Child Adolesc. Psychopharmacol. 16(6), 725-735 (2006).

65. Jung, D. et al. Neuropsychological effects of levetiracetam and carbamazepine in children with focal epilepsy. Neurology 84(23), 2312-2319 (2015).

66. Klein, R. G. Thioridazine effects on the cognitive performance of children with attention-deficit hyperactivity disorder. J. Child Adolesc. Psychopharmacol. 1(4), 263-270 (1990).

67. Kwon, S., Hwang, T. G., Lee, J., Kim, D.-K. \& Seo, H.-E. Benign childhood epilepsy with centrotemporal spikes: To treat or not to treat. J. Epilepsy Res. 3(1), 1 (2013).

68. O’Dougherty, M., Wright, F. S., Cox, S. \& Walson, P. Carbamazepine plasma concentration: Relationship to cognitive impairment. Arch. Neurol. 44(8), 863-867 (1987).

69. Operto, F. F. et al. Effects on executive functions of antiepileptic monotherapy in pediatric age. Epilepsy Behav. 102, 106648 (2020).

70. Pandina, G. J., Zhu, Y. \& Cornblatt, B. Cognitive function with long-term risperidone in children and adolescents with disruptive behavior disorder. J Child Adolesc. Psychopharmacol. 19(6), 749-756 (2009).

71. Piccinelli, P. et al. Neuropsychological and behavioural aspects in children and adolescents with idiopathic epilepsy at diagnosis and after 12 months of treatment. Seizure 19(9), 540-546 (2010).

72. Platt, J. E., Campbell, M., Green, W. H., Perry, R. \& Cohen, I. Effects of lithium carbonate and haloperidol on cognition in aggressive hospitalized school-age children. J. Clin. Psychopharmacol. 1(1), 8-13 (1981).

73. Platt, J. E., Campbell, M., Green, W. H. \& Grega, D. M. Cognitive effects of lithium carbonate and haloperidol in treatment-resistant aggressive children. Arch. Gen. Psychiatry 41(7), 657-662 (1984).

74. Rappaport, L. et al. Effects of theophylline on behavior and learning in children with asthma. Am. J. Dis. Child. 143(3), 368-372 (1989).

75. Robles, O. et al. Cognitive efficacy of quetiapine and olanzapine in early-onset first-episode psychosis. Schizophr. Bull. 37(2), 405-415 (2011).

76. Schlieper, A., Alcock, D., Beaudry, P., Feldman, W. \& Leikin, L. Effect of therapeutic plasma concentrations of theophylline on behavior, cognitive processing, and affect in children with asthma. J. Pediatr. 118(3), 449-455 (1991).

77. Seidel, W. T. \& Mitchell, W. G. Cognitive and behavioral effects of carbamazepine in children: data from benign rolandic epilepsy. J. Child Neurol. 14(11), 716-723 (1999).

78. Shehab, A. A. S., Brent, D. \& Maalouf, F. T. Neurocognitive changes in selective serotonin reuptake inhibitors-treated adolescents with depression. J. Child Adolesc. Psychopharmacol. 26(8), 713-720 (2016).

79. Sommer, B. R., O’Hara, R., Askari, N., Kraemer, H. C. \& Kennedy, W. A. The effect of oxybutynin treatment on cognition in children with diurnal incontinence. J. Urol. 173(6), 2125-2127 (2005).

80. Stevenson, J. et al. On behalf of the Etac Study G: Long-term evaluation of the impact of the H1-receptor antagonist cetirizine on the behavioral, cognitive, and psychomotor development of very young children with atopic dermatitis. Pediatr. Res. 52(2), 251-257 (2002). 
81. Tonnby, B. et al. Withdrawal of antiepileptic medication in children correlation of cognitive function and plasma concentrationThe multicentre 'Holmfrid'study. Epilepsy Res. 19(2), 141-152 (1994).

82. Troost, P. W. et al. Neuropsychological effects of risperidone in children with pervasive developmental disorders: ABlinded discontinuation study. J. Child Adolesc. Psychopharmacol. 16(5), 561-573 (2006).

83. Tzitiridou, M. et al. Oxcarbazepine monotherapy in benign childhood epilepsy with centrotemporal spikes: A clinical and cognitive evaluation. Epilepsy Behav. 7(3), 458-467 (2005).

84. Werry, J. S., Dowrick, P. W., Lampen, E. L. \& Vamos, M. J. Imipramine in enuresis-psychological and physiological effects. J. Child Psychol. Psychiatry 16(4), 289-299 (1975).

85. Wilson, H. \& Staton, R. D. Neuropsychological changes in children associated with tricyclic antidepressant therapy. Int. J. Neurosci. 24(3-4), 307-312 (1984).

86. Yepes, L., Balka, E. B., Winsberg, B. G. \& Bialer, I. Amitriptyline and methylphenidate treatment of behaviorally disordered children. J. Child Psychol. Psychiatry 18(1), 39-52 (1977).

87. Yuan, J. et al. Lithium treatment is safe in children with intellectual disability. Front. Mol. Neurosci. 11, 425 (2018).

88. Cai, X., Campbell, N., Khan, B., Callahan, C. \& Boustani, M. Long-term anticholinergic use and the aging brain. Alzheimer Dement. 9(4), 377-385 (2013).

89. Carrière, I. et al. Drugs with anticholinergic properties, cognitive decline, and dementia in an elderly general population: The 3-city study. Arch. Intern. Med. 169(14), 1317-1324 (2009).

90. Kalisch Ellett, L. M., Pratt, N. L., Ramsay, E. N., Barratt, J. D. \& Roughead, E. E. Multiple anticholinergic medication use and risk of hospital admission for confusion or dementia. J. Am. Geriatr. Soc. 62(10), 1916-1922 (2014).

91. Jack, C. R. Jr. et al. Tracking pathophysiological processes in Alzheimer's disease: An updated hypothetical model of dynamic biomarkers. Lancet Neurol. 12(2), 207-216 (2013).

92. Rosenblat, J. D., Kakar, R. \& McIntyre, R. S. The cognitive effects of antidepressants in major depressive disorder: A systematic review and meta-analysis of randomized clinical trials. Int. J. Neuropsychopharmacol. 19, 2 (2015).

93. Ziad, A. et al. Anticholinergic drug use and cognitive performances in middle age: Findings from the CONSTANCES cohort. J. Neurol. Neurosurg. Psychiatry 89(10), 1107-1115 (2018).

94. Farrall, A. J. \& Wardlaw, J. M. Blood-brain barrier: Ageing and microvascular disease: Systematic review and meta-analysis. Neurobiol. Aging 30(3), 337-352 (2009).

95. Popescu, B. O. et al. Blood-brain barrier alterations in ageing and dementia. J. Neurol. Sci. 283(1), 99-106 (2009).

96. Lezak, M. D. et al. (eds) Neuropsychological Assessment (Oxford University Press, Oxford, 2012).

\title{
Acknowledgement
}

HADK is supported by a NHMRC Dementia Research Leadership Fellowship (GNT1135676). LMKE is supported by an NHMRC-ARC Dementia Research Development Fellowship (APP1101788). KR is supported by the UK Alzheimer's Society.

\section{Author contributions}

H.A.D.K. and L.M.E.K. conceptualised the study in consultation with K.R. H.A.D.K. and M.C. wrote the first draft of the manuscript. E.G. carried out the statistical analyses. M.C., T.J.R., D.C., C.S. and J.N.H. carried out the systematic review. All authors provided intellectual input to the manuscript and approved the final version for submission.

\section{Competing interests}

The authors declare no competing interests.

Additional information

Supplementary Information The online version contains supplementary material available at https://doi. org/10.1038/s41598-020-80211-6.

Correspondence and requests for materials should be addressed to H.A.D.K.

Reprints and permissions information is available at www.nature.com/reprints.

Publisher's note Springer Nature remains neutral with regard to jurisdictional claims in published maps and institutional affiliations.

\begin{abstract}
Open Access This article is licensed under a Creative Commons Attribution 4.0 International License, which permits use, sharing, adaptation, distribution and reproduction in any medium or format, as long as you give appropriate credit to the original author(s) and the source, provide a link to the Creative Commons licence, and indicate if changes were made. The images or other third party material in this article are included in the article's Creative Commons licence, unless indicated otherwise in a credit line to the material. If material is not included in the article's Creative Commons licence and your intended use is not permitted by statutory regulation or exceeds the permitted use, you will need to obtain permission directly from the copyright holder. To view a copy of this licence, visit http://creativecommons.org/licenses/by/4.0/.
\end{abstract}

(c) The Author(s) 2021 